\title{
On Fractional Volterra Integrodifferential Equations with Fractional Integrable Impulses
}

\author{
Sagar T. Sutar ${ }^{a}$ and Kishor D. Kucche ${ }^{b}$ \\ ${ }^{a}$ Department of Mathematics, Vivekanand College (Autonomous) \\ Kolhapur-416003, Maharashtra, India \\ ${ }^{b}$ Department of Mathematics, Shivaji University \\ Kolhapur-416004, Maharashtra, India \\ E-mail(corresp.): sutar.sagar007@gmail.com \\ E-mail: kdkucche@gmail.com
}

Received January 7, 2019; revised May 17, 2019; accepted May 20, 2019

\begin{abstract}
We consider a class of nonlinear fractional Volterra integrodifferential equation with fractional integrable impulses and investigate the existence and uniqueness results in the Bielecki's normed Banach spaces. Further, Bielecki-Ulam type stabilities have been demonstrated on a compact interval. A concrete example is provided to illustrate the outcomes we acquired.
\end{abstract}

Keywords: fractional Volterra integrodifferential equation, integrable impulses, Banach contraction principle, existence of solutions, Bielecki norm, Bielecki-Ulam type stability.

AMS Subject Classification: 34A37; 45M10; 34G20; 34A08.

\section{Introduction}

Famous "Ulam stability problem" of functional differential equation raised by Ulam [10] have been extended to different kinds of equations. Wang et al. [13] are the first mathematicians who investigated the Ulam stability and data dependence for fractional differential equations. Thereafter, several interesting works on different Ulam type stabilities of fractional differential and integral equations have been reported (see for instance [3], [14], [11], [18], [19]).

An overview pertaining to impulsive differential equations with instantaneous impulses and its applicability in the practical dynamical systems have provided in the monograph $[1,2,9]$. The impulsive differential equations with time variable impulses dealt in an interesting papers $[4,5,6]$. Wang et al. $[12,17]$

Copyright (C) 2019 The Author(s). Published by VGTU Press

This is an Open Access article distributed under the terms of the Creative Commons Attribution License (http://creativecommons.org/licenses/by/4.0/), which permits unrestricted use, distribution, and reproduction in any medium, provided the original author and source are credited. 
studied existence and uniqueness of solutions and established generalized $\beta$ Ulam-Hyers-Rassias stability to differential equations with non instantaneous impulses in a $P \beta$-normed Banach space. Zada et al. $[15,20,21,22,23,24,25]$ investigated Ulam-types stabilities for various classes of differential equations with and without impulse effect.

Recently, Wang and Zang [16] introduced a new class of impulsive differential equations of the form

$$
\left\{\begin{array}{l}
x^{\prime}(\tau)=f(\tau, x(\tau)), \tau \in\left(\sigma_{i}, \tau_{i+1}\right], i=0,1, \ldots, m \\
x(\tau)=I_{\tau_{i}, \tau}^{\beta} h_{i}(\tau, x(\tau)), \tau \in\left(\tau_{i}, \sigma_{i}\right], i=1,2, \ldots, m, \beta \in(0,1)
\end{array}\right.
$$

and examined the existence and uniqueness of solutions in Bielecki's normed Banach spaces. Further, demonstrated that the corresponding equations are Bielecki-Ulam-Hyer stable. It is seen that such sort of formulations are adequate to depict the memory procedures of the drugs in the circulation system and the subsequent absorption for the body.

Motivated by the work of Wang and Zang [16], we consider the following class of nonlinear fractional Volterra integrodifferential equation with fractional order integrable impulses of the form

$$
\left\{\begin{array}{c}
{ }_{\sigma_{i}}^{c} \mathcal{D}_{\tau}^{\alpha} x(\tau)=f\left(\tau, x(\tau), \int_{\sigma_{i}}^{\tau} h(\sigma, x(\sigma)) d \sigma\right), \tau \in\left(\sigma_{i}, \tau_{i+1}\right] \\
i=0,1, \ldots, m, \quad \alpha \in(0,1) \\
x(\tau)=\mathcal{I}_{\tau_{i}, \tau}^{\beta} h_{i}(\tau, x(\tau)), \tau \in\left(\tau_{i}, \sigma_{i}\right], i=1,2, \ldots, m, \beta \in(0,1)
\end{array}\right.
$$

and research the existence and uniqueness of solutions and examine the outcomes relating to Bielecki-Ulam type stabilities viz. Bielecki-Ulam-Hyers and Bielecki-Ulam-Hyers-Rassias stabilities on a compact interval. Here $\tau_{i}$ and $\sigma_{i}$ are pre-fixed numbers satisfying $0=\tau_{0}=\sigma_{0}<\tau_{1} \leq \sigma_{1} \leq \tau_{2}<\ldots<\tau_{m} \leq$ $\sigma_{m}<\tau_{m+1}=T, f:[0, T] \times \mathbb{R} \times \mathbb{R} \rightarrow \mathbb{R}, h:[0, T] \times \mathbb{R} \rightarrow \mathbb{R}$ and for each $i=1,2 \ldots m, h_{i}:\left(\tau_{i}, \sigma_{i}\right] \times \mathbb{R} \rightarrow \mathbb{R}$ is continuous function, ${ }_{\sigma_{i}}^{c} \mathcal{D}_{\tau}^{\alpha}$ is the Caputo fractional derivative of order $\alpha$ with lower terminal at $\sigma_{i}$ and $\mathcal{I}_{\tau_{i}, \tau}^{\beta}$ is the Riemann-Liouville fractional integral of order $\beta$ with lower terminal $\tau_{i}$.

We comment that within this scope, the class of equations considered in the present paper is more broad and the outcomes acquired are the generalization of the fundamental results obtained by Wang and Zang [16]. We support our main results with the examples.

In Section 2, we introduce some preliminaries and auxiliary lemmas related to fractional calculus. In Section 3, we prove existence and uniqueness results for (1.1) by using Banach contraction principle via Bielecki norm. In Section 4, adopting the idea of Wang and Zang [16] we examine different Bielecki-Ulam's type stability for the problem (1.1). Finally, an example has been provided to illustrate the results we obtained. 


\section{Preliminaries}

Let $J=[0, T]$. Let $C(J, \mathbb{R})=\{x: J \rightarrow \mathbb{R} ; x$ is continuous function $\}$ be the Banach space endowed with a Bielecki norm

$$
\|x\|_{B}=\sup _{\tau \in J} \frac{|x(\tau)|}{e^{\theta \tau}}
$$

where $\theta>0$ is a fixed real number. Let

$$
\begin{gathered}
P C(J, \mathbb{R})=\left\{x: J \rightarrow \mathbb{R}: x \in C\left(\left(\tau_{i}, \tau_{i+1}\right], \mathbb{R}\right), \text { both } x\left(\tau_{i}^{+}\right) \text {and } x\left(\tau_{i}^{-}\right)\right. \text {exists } \\
\text { and } \left.x\left(\tau_{i}\right)=x\left(\tau_{i}^{-}\right), i=0,1, \ldots, m\right\} .
\end{gathered}
$$

If $P C(J, \mathbb{R})$ is endowed with the norm

$$
\|x\|_{P B}=\sup _{\tau \in J} \frac{|x(\tau)|}{e^{\theta \tau}}, \theta>0,
$$

then $\left(P C(J, \mathbb{R}),\|\cdot\|_{P B}\right)$ is a Banach space. The space

$$
P C^{1}(J, \mathbb{R})=\left\{x \in P C(J, \mathbb{R}): x^{\prime} \in P C(J, \mathbb{R})\right\}
$$

with the norm $\|x\|_{P B^{\prime}}=\max \left\{\|x\|_{P B},\left\|x^{\prime}\right\|_{P B}\right\}$ then $\left(P C^{1}(J, \mathbb{R}),\|\cdot\| P B^{\prime}\right)$ is a Banach space.

Next, we use definitions and the results listed bellow from fractional calculus. For more details, we refer the readers to the monograph [7].

Definition 1 . Let $g \in C[a, T]$ with $T>a \geq 0$ and $\beta \geq 0$, then the RiemannLiouville fractional integral $\mathcal{I}_{a, \tau}^{\beta}$ of order $\beta$ of a function $g$ is defined as

$$
\mathcal{I}_{a, \tau}^{\beta} g(\tau)=\frac{1}{\Gamma(\beta)} \int_{a}^{\tau}(\tau-\sigma)^{\beta-1} g(\sigma) d \sigma, \quad \tau>a \geq 0,
$$

provided the integral exists.

Definition 2. Let $0<\alpha \leq 1$ then the Caputo fractional derivative ${ }_{a}^{c} \mathcal{D}_{\tau}^{\alpha}$ of order $\alpha$ with lower terminal $a$ of a function $g \in C^{1}[a, T]$ is defined as

$$
{ }_{a}^{c} \mathcal{D}_{\tau}^{\alpha} g(\tau)=\frac{1}{\Gamma(1-\alpha)} \int_{a}^{\tau}(\tau-\sigma)^{-\alpha} g^{\prime}(\sigma) d \sigma, \quad \tau>a \geq 0 .
$$

Lemma 1. Let $m-1<\alpha \leq m \in \mathbb{N}$ and $g \in C^{m}[a, T]$. Then

$$
\mathcal{I}_{a, \tau}^{\alpha}\left[{ }_{a}^{c} \mathcal{D}_{\tau}^{\alpha} g(\tau)\right]=g(\tau)-\sum_{k=0}^{m-1} \frac{g^{(k)}(a)}{\Gamma(k+1)} \tau^{k}, \quad \tau>a \geq 0 .
$$

The following lemma plays an important role to obtain our results. 
Lemma 2. [8] Let $\alpha, \beta, \gamma$ and $p$ be constants such that $\alpha>0, p(\gamma-1)+1>0$ and $p(\beta-1)+1>0$. Then

$\int_{0}^{\tau}\left(\tau^{\alpha}-\sigma^{\alpha}\right)^{p(\beta-1)} \sigma^{p(\gamma-1)} d \sigma=\frac{\tau^{\theta}}{\alpha} \mathbb{B}\left(\frac{p(\gamma-1)+1}{\alpha}, p(\beta-1)+1\right), \quad \forall \tau \in \mathbb{R}_{+}$,

where

$$
\mathbb{B}(\xi, \sigma)=\int_{0}^{1} \sigma^{\xi-1}(1-\sigma)^{\sigma-1} d \sigma, \quad \operatorname{Re}(\xi)>0, \operatorname{Re}(\sigma)>0
$$

is a Beta function and $\theta=p[\alpha(\beta-1)+\gamma-1]+1$.

\section{Existence and uniqueness results}

Lemma 3. A function $x \in P C^{1}(J, \mathbb{R})$ is a classical solution of the problem

$$
\left\{\begin{array}{c}
{ }_{\sigma_{i}}^{c} \mathcal{D}_{\tau}^{\alpha} x(\tau)=f\left(\tau, x(\tau), \int_{\sigma_{i}}^{\tau} h(\sigma, x(\sigma)) d \sigma\right), \tau \in\left(\sigma_{i}, \tau_{i+1}\right], \\
i=0,1, \ldots, m, \quad \alpha \in(0,1), \\
x(\tau)=\mathcal{I}_{\tau_{i}, \tau}^{\beta} h_{i}(\tau, x(\tau)), \tau \in\left(\tau_{i}, \sigma_{i}\right], i=1,2, \ldots, m, \beta \in(0,1), \\
x(0)=x_{0},
\end{array}\right.
$$

if $x$ satisfies the fractional Volterra integral equations

$$
x(\tau)=\left\{\begin{array}{l}
x_{0}, \tau=0, \\
\mathcal{I}_{\tau_{i}, \tau}^{\beta} h_{i}(\tau, x(\tau)), \tau \in\left(\tau_{i}, \sigma_{i}\right], i=1, \ldots, m, \\
x_{0}+\mathcal{I}_{0, \tau}^{\alpha} f\left(\tau, x(\tau), \int_{0}^{\tau} h(\sigma, x(\sigma)) d \sigma\right), \tau \in\left(0, \tau_{1}\right], \\
\mathcal{I}_{\tau_{i}, \sigma_{i}}^{\beta} h_{i}\left(\sigma_{i}, x\left(\sigma_{i}\right)\right)+\mathcal{I}_{\sigma_{i}, \tau}^{\alpha} f\left(\tau, x(\tau), \int_{\sigma_{i}}^{\tau} h(\sigma, x(\sigma)) d \sigma\right), \\
\tau \in\left(\sigma_{i}, \tau_{i+1}\right], i=1, \ldots, m .
\end{array}\right.
$$

Proof. For $i=0$, on operating Riemann-Liouville fractional integral operator $\mathcal{I}_{\sigma_{i}, \tau}^{\alpha}$ on both sides of fractional differential equation (3.1), we get

$$
\mathcal{I}_{0, \tau}^{\alpha}\left[{ }_{0}^{c} \mathcal{D}_{\tau}^{\alpha} x(\tau)\right]=\mathcal{I}_{0, \tau}^{\alpha} f\left(\tau, x(\tau), \int_{0}^{\tau} h(\sigma, x(\sigma)) d \sigma\right), \tau \in\left(0, \tau_{1}\right] .
$$

As $0<\alpha<1$, in view of Lemma 1 , we obtain

$$
x(\tau)-x(0)=\mathcal{I}_{0, \tau}^{\alpha} f\left(\tau, x(\tau), \int_{0}^{\tau} h(\sigma, x(\sigma)) d \sigma\right), \tau \in\left(0, \tau_{1}\right] .
$$

Therefore

$$
x(\tau)=x_{0}+\mathcal{I}_{0, \tau}^{\alpha} f\left(\tau, x(\tau), \int_{0}^{\tau} h(\sigma, x(\sigma)) d \sigma\right), \quad \tau \in\left(0, \tau_{1}\right] .
$$

Similarly, for each $i(i=1,2, \ldots, m)$, operating $\mathcal{I}_{\sigma_{i}, \tau}^{\alpha}$ on both sides of $(3.1)$, we get

$$
x(\tau)=x\left(\sigma_{i}\right)+\mathcal{I}_{\sigma_{i}, \tau}^{\alpha} f\left(\tau, x(\tau), \int_{\sigma_{i}}^{\tau} h(\sigma, x(\sigma)) d \sigma\right), \quad \tau \in\left(\sigma_{i}, \tau_{i+1}\right] .
$$


But from equation (3.1), we have

$$
x\left(\sigma_{i}\right)=\left[\mathcal{I}_{\tau_{i}, \tau}^{\beta} h_{i}(\tau, x(\tau))\right]_{\tau=\sigma_{i}}=\mathcal{I}_{\tau_{i}, \sigma_{i}}^{\beta} h_{i}\left(\sigma_{i}, x\left(\sigma_{i}\right)\right), \quad i=1,2, \ldots, m .
$$

Therefore

$$
x(\tau)=\mathcal{I}_{\tau_{i}, \sigma_{i}}^{\beta} h_{i}\left(\sigma_{i}, x\left(\sigma_{i}\right)\right)+\mathcal{I}_{\sigma_{i}, \tau}^{\alpha} f\left(\tau, x(\tau), \int_{\sigma_{i}}^{\tau} h(\sigma, x(\sigma)) d \sigma\right), \quad \tau \in\left(\sigma_{i}, \tau_{i+1}\right] .
$$

We list the following hypotheses in order to establish our main results.

(H1) The function $f \in C(J \times \mathbb{R} \times \mathbb{R}, \mathbb{R})$ satisfies the Lipschitz condition

$$
|f(\tau, x, y)-f(\tau, \bar{x}, \bar{y})| \leq M_{f}|x-\bar{x}|+N_{f}|y-\bar{y}|, \tau \in J ; x, \bar{x}, y, \bar{y} \in \mathbb{R}
$$

where $M_{f}>0, N_{f}>0$.

(H2) The function $h \in C(J \times \mathbb{R}, \mathbb{R})$ satisfies the Lipschitz condition

$$
|h(\tau, x)-h(\tau, \bar{x})| \leq K_{h}|x-\bar{x}|, \tau \in J ; x, \bar{x} \in \mathbb{R}
$$

where $K_{h}>0$.

(H3) For each $i=1,2, \ldots, m ; h_{i} \in C\left(\left(\sigma_{i}, \tau_{i}\right] \times \mathbb{R}, \mathbb{R}\right)$ and satisfies Lipschitz condition

$$
\left|h_{i}(\tau, x)-h_{i}(\tau, \bar{x})\right| \leq L_{h_{i}}|x-\bar{x}|, \text { for each } \tau \in\left(\sigma_{i}, \tau_{i}\right] ; x, \bar{x} \in \mathbb{R}
$$

where $L_{h_{i}}>0$.

Theorem 1. Assume that hypotheses (H1)-(H3) hold. Then the problem (3.1) has a unique solution, provided that $\alpha, \beta \in\left(\frac{1}{2}, 1\right)$.

Proof. Define an operator $T: P C(J, \mathbb{R}) \rightarrow P C(J, \mathbb{R})$ by

$$
(T x)(\tau)=\left\{\begin{array}{l}
x_{0}, \text { if } \tau=0, \\
\mathcal{I}_{\tau_{i}, \tau}^{\beta} h_{i}(\tau, x(\tau)), \text { if } \tau \in\left(\tau_{i}, \sigma_{i}\right], i=1, \ldots, m, \\
x_{0}+\mathcal{I}_{0, \tau}^{\alpha} f\left(\tau, x(\tau), \int_{0}^{\tau} h(\sigma, x(\sigma)) d \sigma\right), \text { if } \tau \in\left(0, \tau_{1}\right], \\
\mathcal{I}_{\tau_{i}, \sigma_{i}}^{\beta} h_{i}\left(\sigma_{i}, x\left(\sigma_{i}\right)\right)+\mathcal{I}_{\sigma_{i}, \tau}^{\alpha} f\left(\tau, x(\tau), \int_{\sigma_{i}}^{\tau} h(\sigma, x(\sigma)) d \sigma\right), \\
\text { if } \tau \in\left(\sigma_{i}, \tau_{i+1}\right], i=1, \ldots, m .
\end{array}\right.
$$

We shall show that the operator $T$ is contraction with respect to Bielecki norm. Let $x, y \in P C(J, \mathbb{R})$ and $\tau \in\left(\tau_{i}, \sigma_{i}\right], i=1,2, \ldots, m$, then using hypothesis 
(H3), we have

$$
\begin{aligned}
& \left|\mathcal{I}_{\tau_{i}, \tau}^{\beta} h_{i}(\tau, x(\tau))-\mathcal{I}_{\tau_{i}, \tau}^{\beta} h_{i}(\tau, y(\tau))\right| \\
& =\left|\frac{1}{\Gamma(\beta)} \int_{\tau_{i}}^{\tau}(\tau-\sigma)^{\beta-1} h_{i}(\sigma, x(\sigma)) d \sigma-\frac{1}{\Gamma(\beta)} \int_{\tau_{i}}^{\tau}(\tau-\sigma)^{\beta-1} h_{i}(\sigma, y(\sigma)) d \sigma\right| \\
& \leq \frac{1}{\Gamma(\beta)} \int_{\tau_{i}}^{\tau}(\tau-\sigma)^{\beta-1}\left|h_{i}(\sigma, x(\sigma))-h_{i}(\sigma, y(\sigma))\right| d \sigma \\
& \leq \frac{L_{h_{i}}}{\Gamma(\beta)} \int_{\tau_{i}}^{\tau}(\tau-\sigma)^{\beta-1}|x(\sigma)-y(\sigma)| d \sigma \\
& \leq \frac{L_{h_{i}}}{\Gamma(\beta)} \int_{\tau_{i}}^{\tau}(\tau-\sigma)^{\beta-1} e^{\theta \sigma}\left(\sup _{\sigma \in\left(\tau_{i}, \sigma_{i}\right]} e^{-\theta \sigma}|x(\sigma)-y(\sigma)|\right) d \sigma \\
& \leq \frac{L_{h_{i}}}{\Gamma(\beta)}\|x-y\|_{P B} \int_{\tau_{i}}^{\tau}(\tau-\sigma)^{\beta-1} e^{\theta \sigma} d \sigma .
\end{aligned}
$$

By Hölders inequality, for $\beta \in\left(\frac{1}{2}, 1\right)$ and $\tau \in\left(\tau_{i}, \sigma_{i}\right]$, we have

$$
\begin{aligned}
& \int_{\tau_{i}}^{\tau}(\tau-\sigma)^{\beta-1} e^{\theta \sigma} d \sigma \leq\left(\int_{\tau_{i}}^{\tau}(\tau-\sigma)^{2(\beta-1)} d \sigma\right)^{\frac{1}{2}}\left(\int_{\tau_{i}}^{\tau} e^{2 \theta \sigma} d \sigma\right)^{\frac{1}{2}} \\
& =\left(\frac{\left(\tau-\tau_{i}\right)^{2 \beta-1}}{2 \beta-1}\right)^{\frac{1}{2}}\left(\frac{e^{2 \theta \tau}-e^{2 \theta \tau_{i}}}{2 \theta}\right)^{\frac{1}{2}} \leq \frac{\left(\sigma_{i}-\tau_{i}\right)^{\beta-\frac{1}{2}}}{(2 \beta-1)^{\frac{1}{2}}}\left(\frac{e^{\theta \tau}-e^{\theta \tau_{i}}}{(2 \theta)^{\frac{1}{2}}}\right) \\
& \leq \frac{\left(\sigma_{i}-\tau_{i}\right)^{\beta-\frac{1}{2}}}{(2 \beta-1)^{\frac{1}{2}}(2 \theta)^{\frac{1}{2}}} e^{\theta \tau} .
\end{aligned}
$$

Thus for any $x, y \in P C(J, \mathbb{R})$ and $\tau \in\left(\tau_{i}, \sigma_{i}\right], i=1,2, \ldots, m$, we have

$$
\left|\mathcal{I}_{\tau_{i}, \tau}^{\beta} h_{i}(\tau, x(\tau))-\mathcal{I}_{\tau_{i}, \tau}^{\beta} h_{i}(\tau, y(\tau))\right| \leq \frac{L_{h_{i}}}{\Gamma(\beta)} \frac{\left(\sigma_{i}-\tau_{i}\right)^{\beta-\frac{1}{2}}}{(2 \beta-1)^{\frac{1}{2}}(2 \theta)^{\frac{1}{2}}} e^{\theta \tau}\|x-y\|_{P B} .
$$

Now, for any $x, y \in P C(J, \mathbb{R})$ and $\tau \in\left(\sigma_{i}, \tau_{i+1}\right], i=1,2, \ldots, m$ by using hypotheses (H1)-(H2), we obtain

$$
\begin{aligned}
\mid \mathcal{I}_{\sigma_{i}, \tau}^{\alpha} & f\left(\tau, x(\tau), \int_{\sigma_{i}}^{\tau} h(\sigma, x(\sigma)) d \sigma\right)-\mathcal{I}_{\sigma_{i}, \tau}^{\alpha} f\left(\tau, y(\tau), \int_{\sigma_{i}}^{\tau} h(\sigma, y(\sigma)) d \sigma\right) \mid \\
& \leq \mathcal{I}_{\sigma_{i}, \tau}^{\alpha} \mid f\left(\tau, x(\tau), \mathcal{I}_{\sigma_{i}, \tau}^{1} h(\tau, x(\tau))-f\left(\tau, y(\tau), \mathcal{I}_{\sigma_{i}, \tau}^{1} h(\tau, y(\tau)) \mid\right.\right. \\
& \leq \mathcal{I}_{\sigma_{i}, \tau}^{\alpha}\left(M_{f}|x(\tau)-y(\tau)|+N_{f}\left|\mathcal{I}_{\sigma_{i}, \tau}^{1} h(\tau, x(\tau))-\mathcal{I}_{\sigma_{i}, \tau}^{1} h(\tau, y(\tau))\right|\right) \\
& \leq \mathcal{I}_{\sigma_{i}, \tau}^{\alpha}\left(M_{f}|x(\tau)-y(\tau)|+N_{f} \mathcal{I}_{\sigma_{i}, \tau}^{1}|h(\tau, x(\tau))-h(\tau, y(\tau))|\right) \\
& \left.\leq \mathcal{I}_{\sigma_{i}, \tau}^{\alpha}\left(M_{f}|x(\tau)-y(\tau)|+N_{f} \mathcal{I}_{\sigma_{i}, \tau}^{1} K_{h} \mid x(\tau)-y(\tau)\right) \mid\right) \\
& =M_{f} \mathcal{I}_{\sigma_{i}, \tau}^{\alpha}|x(\tau)-y(\tau)|+N_{f} K_{h} \mathcal{I}_{\sigma_{i}, \tau}^{\alpha}\left(\mathcal{I}_{\sigma_{i}, \tau}^{1}|x(\tau)-y(\tau)|\right) \\
& =M_{f} \mathcal{I}_{\sigma_{i}, \tau}^{\alpha}|x(\tau)-y(\tau)|+N_{f} K_{h} \mathcal{I}_{\sigma_{i}, \tau}^{\alpha+1}|x(\tau)-y(\tau)| \\
= & \frac{M_{f}}{\Gamma(\alpha)} \int_{\sigma_{i}}^{\tau}(\tau-\sigma)^{\alpha-1}|x(\sigma)-y(\sigma)| d \sigma+\frac{N_{f} K_{h}}{\Gamma(\alpha+1)} \int_{\sigma_{i}}^{\tau}(\tau-\sigma)^{\alpha}|x(\sigma)-y(\sigma)| d \sigma
\end{aligned}
$$




$$
\begin{aligned}
= & \frac{M_{f}}{\Gamma(\alpha)} \int_{\sigma_{i}}^{\tau}(\tau-\sigma)^{\alpha-1} e^{\theta \sigma}\left(\sup _{\sigma \in\left(\sigma_{i}, \tau_{i+1}\right]} e^{-\theta \sigma}|x(\sigma)-y(\sigma)|\right) d \sigma+\frac{N_{f} K_{h}}{\Gamma(\alpha+1)} \\
& \times \int_{\sigma_{i}}^{\tau}(\tau-\sigma)^{\alpha}|x(\sigma)-y(\sigma)| e^{\theta \sigma}\left(\sup _{\sigma \in\left(\sigma_{i}, \tau_{i+1}\right]} e^{-\theta \sigma}|x(\sigma)-y(\sigma)|\right) d \sigma \\
\leq & \frac{M_{f}}{\Gamma(\alpha)} \times\|x-y\|_{P B} \int_{\sigma_{i}}^{\tau}(\tau-\sigma)^{\alpha-1} e^{\theta \sigma} d \sigma+\frac{N_{f} K_{h}}{\Gamma(\alpha+1)}\|x-y\|_{P B} \int_{\sigma_{i}}^{\tau}(\tau-\sigma)^{\alpha} e^{\theta \sigma} d \sigma .
\end{aligned}
$$

Since $\alpha \in\left(\frac{1}{2}, 1\right)$, from the inequality $(3.2)$, for any $\tau \in\left(\sigma_{i}, \tau_{i+1}\right], i=1,2, \ldots, m$, we have

$$
\int_{\sigma_{i}}^{\tau}(\tau-\sigma)^{\alpha-1} e^{\theta \sigma} d \sigma \leq \frac{\left(\tau-\sigma_{i}\right)^{\alpha-\frac{1}{2}}}{(2 \alpha-1)^{\frac{1}{2}}(2 \theta)^{\frac{1}{2}}} e^{\theta \tau} \leq \frac{\left(\tau_{i+1}-\sigma_{i}\right)^{\alpha-\frac{1}{2}}}{(2 \alpha-1)^{\frac{1}{2}}(2 \theta)^{\frac{1}{2}}} e^{\theta \tau} .
$$

By replacing $\alpha$ by $(\alpha+1)$ in the above inequality, we get

$$
\int_{\sigma_{i}}^{\tau}(\tau-\sigma)^{\alpha} e^{\theta \sigma} d \sigma \leq \frac{\left(\tau_{i+1}-\sigma_{i}\right)^{\alpha+\frac{1}{2}}}{(2 \alpha+1)^{\frac{1}{2}}(2 \theta)^{\frac{1}{2}}} e^{\theta \tau}, \quad \tau \in\left(\sigma_{i}, \tau_{i+1}\right], i=1,2, \ldots, m
$$

Using the inequalities (3.5) and (3.6), the inequality (3.4) takes the form

$$
\begin{aligned}
& \left|\mathcal{I}_{\sigma_{i}, \tau}^{\alpha} f\left(\tau, x(\tau), \int_{\sigma_{i}}^{\tau} h(\sigma, y(\sigma)) d \sigma\right)-\mathcal{I}_{\sigma_{i}, \tau}^{\alpha} f\left(\tau, y(\tau), \int_{\sigma_{i}}^{\tau} h(\sigma, y(\sigma)) d \sigma\right)\right| \\
& \leq\left[\frac{M_{f}}{\Gamma(\alpha)} \frac{\left(\tau_{i+1}-\sigma_{i}\right)^{\alpha-\frac{1}{2}}}{(2 \alpha-1)^{\frac{1}{2}}(2 \theta)^{\frac{1}{2}}}+\frac{N_{f} K_{h}}{\Gamma(\alpha+1)} \frac{\left(\tau_{i+1}-\sigma_{i}\right)^{\alpha+\frac{1}{2}}}{(2 \alpha+1)^{\frac{1}{2}}(2 \theta)^{\frac{1}{2}}}\right] e^{\theta \tau}\|x-y\|_{P B} .
\end{aligned}
$$

From the definition of an operator $T$ and hypothesis (H1)-(H3), for any $x, y \in$ $P C(J, \mathbb{R})$, we obtain that

Case 1: For $\tau \in\left(\tau_{i}, \sigma_{i}\right], i=1,2, \ldots, m$, from the inequality (3.3), we have

$$
\begin{aligned}
|T x(\tau)-T y(\tau)| e^{-\theta \tau} & \leq\left|\mathcal{I}_{\tau_{i}, \tau}^{\beta} h_{i}(\tau, x(\tau))-\mathcal{I}_{\tau_{i}, \tau}^{\beta} h_{i}(\tau, y(\tau))\right| e^{-\theta \tau} \\
& \leq \frac{L_{h_{i}}}{\Gamma(\beta)} \frac{\left(\sigma_{i}-\tau_{i}\right)^{\beta-\frac{1}{2}}}{(2 \beta-1)^{\frac{1}{2}}(2 \theta)^{\frac{1}{2}}}\|x-y\|_{P B} .
\end{aligned}
$$

Case 2: For $\tau \in\left(0, \tau_{1}\right]$, on similar line of $(3.7)$, we have

$$
\begin{aligned}
& |T x(\tau)-T y(\tau)| e^{-\theta \tau}=\mid \mathcal{I}_{0, \tau}^{\alpha} f\left(\tau, x(\tau), \int_{0}^{\tau} h(\sigma, x(\sigma)) d \sigma\right) \\
& \quad-\mathcal{I}_{0, \tau}^{\alpha} f\left(\tau, y(\tau), \int_{0}^{\tau} h(\sigma, y(\sigma)) d \sigma\right) \mid e^{-\theta \tau} \\
& \leq\left[\frac{M_{f}}{\Gamma(\alpha)} \frac{\tau_{1}^{\alpha-\frac{1}{2}}}{(2 \alpha-1)^{\frac{1}{2}}(2 \theta)^{\frac{1}{2}}}+\frac{N_{f} K_{h}}{\Gamma(\alpha+1)} \frac{\tau_{1}^{\alpha+\frac{1}{2}}}{(2 \alpha+1)^{\frac{1}{2}}(2 \theta)^{\frac{1}{2}}}\right]\|x-y\|_{P B} .
\end{aligned}
$$


Case 3: For $\tau \in\left(\sigma_{i}, \tau_{i+1}\right], i=1,2, \ldots, m$, using the inequalities (3.3) and (3.7), we have

$$
\begin{aligned}
& |T x(\tau)-T y(\tau)| e^{-\theta \tau}=\left|\mathcal{I}_{\tau_{i}, \sigma_{i}}^{\beta} h_{i}\left(\sigma_{i}, x\left(\sigma_{i}\right)\right)-\mathcal{I}_{\tau_{i}, \sigma_{i}}^{\beta} h_{i}\left(\sigma_{i}, y\left(\sigma_{i}\right)\right)\right| e^{-\theta \tau} \\
& +\left|\mathcal{I}_{\sigma_{i}, \tau}^{\alpha} f\left(\tau, x(\tau), \int_{\sigma_{i}}^{\tau} h(\sigma, x(\sigma)) d \sigma\right)-\mathcal{I}_{\sigma_{i}, t}^{\alpha} f\left(\tau, y(\tau), \int_{\sigma_{i}}^{\tau} h(\sigma, y(\sigma)) d \sigma\right)\right| e^{-\theta \tau} \\
& \leq \frac{L_{h_{i}}}{\Gamma(\beta)} \frac{\left(\sigma_{i}-\tau_{i}\right)^{\beta-\frac{1}{2}}}{(2 \beta-1)^{\frac{1}{2}}(2 \theta)^{\frac{1}{2}}} e^{\theta\left(\sigma_{i}-\tau\right)}\|x-y\|_{P B} \\
& \quad+\left[\frac{M_{f}}{\Gamma(\alpha)} \frac{\left(\tau_{i+1}-\sigma_{i}\right)^{\alpha-\frac{1}{2}}}{(2 \alpha-1)^{\frac{1}{2}}(2 \theta)^{\frac{1}{2}}}+\frac{N_{f} K_{h}}{\Gamma(\alpha+1)} \frac{\left(\tau_{i+1}-\sigma_{i}\right)^{\alpha+\frac{1}{2}}}{(2 \alpha+1)^{\frac{1}{2}}(2 \theta)^{\frac{1}{2}}}\right]\|x-y\|_{P B} \\
& \leq\left(\frac{L_{h_{i}}}{\Gamma(\beta)} \frac{\left(\sigma_{i}-\tau_{i}\right)^{\beta-\frac{1}{2}}}{(2 \beta-1)^{\frac{1}{2}}(2 \theta)^{\frac{1}{2}}}+\frac{M_{f}}{\Gamma(\alpha)} \frac{\left(\tau_{i+1}-\sigma_{i}\right)^{\alpha-\frac{1}{2}}}{(2 \alpha-1)^{\frac{1}{2}}(2 \theta)^{\frac{1}{2}}}\right. \\
& \left.\quad+\frac{N_{f} K_{h}}{\Gamma(\alpha+1)} \frac{\left(\tau_{i+1}-\sigma_{i}\right)^{\alpha+\frac{1}{2}}}{(2 \alpha+1)^{\frac{1}{2}}(2 \theta)^{\frac{1}{2}}}\right)\|x-y\|_{P B} .
\end{aligned}
$$

From Cases 1-3, we can conclude that

$$
\|T x-T y\|_{P B} \leq \mathcal{L}\|x-y\|_{P B}, \text { for any } x, y \in P C(J, \mathbb{R}),
$$

where

$$
\begin{aligned}
\mathcal{L}=\max \left\{\frac{L_{h_{i}}}{\Gamma(\beta)} \frac{\left(\sigma_{i}-\tau_{i}\right)^{\beta-\frac{1}{2}}}{\sqrt{(2 \beta-1)} \sqrt{2 \theta}}+\frac{M_{f}}{\Gamma(\alpha)} \frac{\left(\tau_{i+1}-\sigma_{i}\right)^{\alpha-\frac{1}{2}}}{\sqrt{(2 \alpha-1)} \sqrt{2 \theta}}\right. \\
\left.+\frac{N_{f} K_{h}}{\Gamma(\alpha+1)} \frac{\left(\tau_{i+1}-\sigma_{i}\right)^{\alpha+\frac{1}{2}}}{\sqrt{2 \alpha+1} \sqrt{2 \theta}} ; i=0,1, \ldots, m\right\} .
\end{aligned}
$$

Choose sufficiently large value of $\theta$ so that $\mathcal{L}<1$. Thus, $T$ becomes a contraction mapping and has a unique fixed point due to Banach contraction principle. This fixed point of $T$ act as unique solution of the problem (3.1).

Next, our aim is to extend the restriction of $\alpha, \beta \in\left(\frac{1}{2}, 1\right)$ to $\alpha, \beta \in(0,1)$. For this purpose we use the following variants of the hypotheses (H1) and (H3):

( $\tilde{H} 1)$ The function $f \in C(J \times \mathbb{R} \times \mathbb{R}, \mathbb{R})$ satisfies the Lipschitz condition $|f(\tau, x, y)-f(\tau, \bar{x}, \bar{y})| \leq M_{f} \tau^{\gamma_{f}}|x-\bar{x}|+N_{f}|y-\bar{y}|, \tau \in J ; x, \bar{x}, y, \bar{y} \in \mathbb{R}$, where $M_{f}, N_{f}>0$ and $\gamma_{f}>-\alpha$.

(苂3) For each $i=1,2, \ldots, m ; h_{i} \in C\left(\left(\sigma_{i}, \tau_{i}\right] \times \mathbb{R}, \mathbb{R}\right)$ and satisfies Lipschitz condition

$$
\left|h_{i}(\tau, x)-h_{i}(\tau, \bar{x})\right| \leq \tau^{\gamma} L_{h_{i}}|x-\bar{x}|, \tau \in\left[\tau_{i}, \sigma_{i}\right] ; x, \bar{x} \in \mathbb{R},
$$

where $L_{h_{i}}>0$ and $\gamma>-\beta$. 


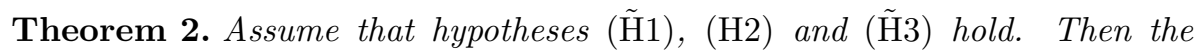
problem (3.1) has a unique solution.

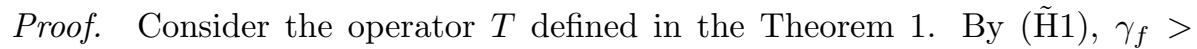
$-\alpha=(1-\alpha)-1$ where $\alpha \in(0,1)$. Choose $\sigma>1$ such that $\sigma \gamma_{f}>\sigma(1-\alpha)-1$ and $\sigma(\alpha-1)+1>0$. Define $\sigma^{*}=\frac{\sigma}{\sigma-1}$. Then $\sigma+\sigma^{*}=1$. By ( $\left.\tilde{\mathrm{H}} 3\right)$, for any $\tau \in\left(\sigma_{i}, \tau_{i+1}\right]$, we have

$$
\begin{aligned}
\mid & \mathcal{I}_{\sigma_{i}, \tau}^{\alpha} f\left(\tau, x(\tau), \int_{\sigma_{i}}^{\tau} h(\sigma, x(\sigma)) d \sigma\right)-\mathcal{I}_{\sigma_{i}, \tau}^{\alpha} f\left(\tau, y(\tau), \int_{\sigma_{i}}^{\tau} h(\sigma, y(\sigma)) d \sigma\right) \mid \\
\leq & \mathcal{I}_{\sigma_{i}, \tau}^{\alpha} \mid f\left(\tau, x(\tau), \mathcal{I}_{\sigma_{i}, \tau}^{1} h(\tau, x(\tau))-f\left(\tau, y(\tau), \mathcal{I}_{\sigma_{i}, \tau}^{1} h(\tau, y(\tau)) \mid\right.\right. \\
\leq & \mathcal{I}_{\sigma_{i}, \tau}^{\alpha}\left(M_{f} \tau^{\gamma_{f}}|x(t)-y(\tau)|+N_{f}\left|\mathcal{I}_{\sigma_{i}, \tau}^{1} h(\tau, x(\tau))-\mathcal{I}_{\sigma_{i}, \tau}^{1} h(\tau, y(\tau))\right|\right) \\
\leq & \mathcal{I}_{\sigma_{i}, \tau}^{\alpha}\left(M_{f} \tau^{\gamma_{f}}|x(\tau)-y(\tau)|+N_{f} \mathcal{I}_{\sigma_{i}, \tau}^{1}|h(\tau, x(\tau))-h(\tau, y(\tau))|\right) \\
\leq & \mathcal{I}_{\sigma_{i}, \tau}^{\alpha}\left(M_{f} \tau^{\gamma_{f}}|x(\tau)-y(\tau)|+N_{f} \mathcal{I}_{\sigma_{i}, \tau} K_{h}|x(\tau)-y(\tau)|\right) \\
= & M_{f} \mathcal{I}_{\sigma_{i}, \tau}^{\alpha} \tau^{\gamma_{f}}|x(\tau)-y(\tau)|+N_{f} K_{h} \mathcal{I}_{\sigma_{i}, \tau}^{\alpha}\left(\mathcal{I}_{\sigma_{i}, \tau}^{1}|x(\tau)-y(\tau)|\right) \\
= & M_{f} \mathcal{I}_{\sigma_{i}, \tau}^{\alpha}\left(\tau^{\gamma_{f}}|x(\tau)-y(\tau)|\right)+N_{f} K_{h} \mathcal{I}_{\sigma_{i}, \tau}^{\alpha+1}(|x(\tau)-y(\tau)|)=\frac{M_{f}}{\Gamma(\alpha)} \\
\times & \int_{\sigma_{i}}^{\tau}(\tau-\sigma)^{\alpha-1} \sigma^{\gamma_{f}}|x(\sigma)-y(\sigma)| d \sigma+\frac{N_{f} K_{h}}{\Gamma(\alpha+1)} \int_{\sigma_{i}}^{\tau}(\tau-\sigma)^{\alpha}|x(\sigma)-y(\sigma)| d \sigma \\
\leq & \frac{M_{f}}{\Gamma(\alpha)} \int_{\sigma_{i}}^{\tau}(\tau-\sigma)^{\alpha-1} \sigma^{\gamma_{f}} e^{\theta \sigma}\left(\sup _{\sigma \in\left(\sigma_{i}, \tau_{i+1}\right]} e^{-\theta \sigma}|x(\sigma)-y(\sigma)|\right) d \sigma \\
& +\frac{N_{f} K_{h}}{\Gamma(\alpha+1)} \int_{\sigma_{i}}^{\tau}(\tau-\sigma)^{\alpha}|x(\sigma)-y(\sigma)| e^{\theta \sigma}\left(\sup _{\sigma \in\left(\sigma_{i}, \tau_{i+1}\right]} e^{-\theta \sigma}|x(\sigma)-y(\sigma)|\right) d \sigma \\
\leq & \frac{M_{f}}{\Gamma(\alpha)}\|x-y\|_{P B} \int_{\sigma_{i}}^{\tau}(\tau-\sigma)^{\alpha-1} \sigma^{\gamma_{f}} e^{\theta \sigma} d \sigma \\
& +\frac{N_{f} K_{h}}{\Gamma(\alpha+1)}\|x-y\|_{P B} \int_{\sigma_{i}}^{\tau}(\tau-\sigma)^{\alpha} e^{\theta \sigma} d \sigma .
\end{aligned}
$$

Using Hölders inequality, we get

$$
\int_{\sigma_{i}}^{\tau}(\tau-\sigma)^{\alpha-1} \sigma^{\gamma_{f}} e^{\theta \sigma} d \sigma \leq\left(\int_{\sigma_{i}}^{\tau}(\tau-\sigma)^{\sigma(\alpha-1)} \sigma^{\sigma \gamma_{f}} d \sigma\right)^{\frac{1}{\sigma}}\left(\int_{\sigma_{i}}^{\tau} e^{\theta \sigma^{*} \sigma} d \sigma\right)^{\frac{1}{\sigma^{*}}} .
$$

But

$$
\left(\int_{\sigma_{i}}^{\tau} e^{\theta \sigma^{*} \sigma} d \sigma\right)^{\frac{1}{\sigma^{*}}}=\left(\frac{e^{\theta \sigma^{*} \tau}-e^{\theta \sigma^{*} \sigma_{i}}}{\theta \sigma^{*}}\right)^{\frac{1}{\sigma^{*}}} \leq\left(\frac{e^{\theta \sigma^{*} \tau}}{\theta \sigma^{*}}\right)^{\frac{1}{\sigma^{*}}}=\frac{e^{\theta \tau}}{\left(\theta \sigma^{*}\right)^{\frac{1}{\sigma^{*}}}} .
$$

By Lemma 2,

$$
\begin{aligned}
& \left(\int_{\sigma_{i}}^{\tau}(\tau-\sigma)^{\sigma(\alpha-1)} \sigma^{\sigma \gamma_{f}} d \sigma\right)^{1 / \sigma} \leq\left(\int_{0}^{\tau}(\tau-\sigma)^{\sigma(\alpha-1)} \sigma^{\sigma \gamma_{f}} d \sigma\right)^{1 / \sigma} \\
& =\left(\tau^{\sigma(\alpha-1)+\sigma \gamma_{f}+1} \mathbb{B}\left(\sigma \gamma_{f}+1, \sigma(\alpha-1)+1\right)\right)^{1 / \sigma} \leq \omega_{1}
\end{aligned}
$$


where $\omega_{1}=\left(T^{\sigma(\alpha-1)+\sigma \gamma_{f}+1} \mathbb{B}\left(\sigma \gamma_{f}+1, \sigma(\alpha-1)+1\right)\right)^{1 / \sigma}$. Therefore,

$$
\int_{\sigma_{i}}^{\tau}(\tau-\sigma)^{\alpha-1} \sigma^{\gamma_{f}} e^{\theta \sigma} d \sigma \leq \omega_{1} \frac{e^{\theta \tau}}{\left(\theta \sigma^{*}\right)^{1 / \sigma^{*}}}
$$

Using equations (3.6) and (3.9), from (3.8) we get

$$
\begin{aligned}
& \left|\mathcal{I}_{\sigma_{i}, \tau}^{\alpha} f\left(\tau, x(\tau), \int_{\sigma_{i}}^{\tau} h(\sigma, y(\sigma)) d \sigma\right)-\mathcal{I}_{\sigma_{i}, \tau}^{\alpha} f\left(\tau, y(\tau), \int_{\sigma_{i}}^{\tau} h(\sigma, y(\sigma)) d \sigma\right)\right| e^{-\theta \tau} \\
& \leq\left(\omega_{1} \frac{M_{f}}{\Gamma(\alpha)\left(\theta \sigma^{*}\right)^{\frac{1}{\sigma^{*}}}}+\frac{N_{f} K_{h}}{\Gamma(\alpha+1)} \frac{\left(\tau_{i+1}-\sigma_{i}\right)^{\alpha+\frac{1}{2}}}{(2 \alpha+1)^{\frac{1}{2}}(2 \theta)^{\frac{1}{2}}}\right)\|x-y\|_{P B} .
\end{aligned}
$$

On the similar line by ( $\tilde{\mathrm{H}} 3)$, as discussed above, we can choose $\sigma_{1}>1$ such that $\sigma_{1} \gamma>\sigma_{1}(1-\beta)-1$ and $\sigma_{1}(\beta-1)+1>0$. Then for $\sigma_{1}^{*}=\frac{\sigma_{1}}{\sigma_{1}-1}$, we have $\sigma_{1}+\sigma_{1}^{*}=1$. By $(\tilde{\mathrm{H}} 3)$ and the Hölders inequality, for any $\tau \in\left(\tau_{i}, \sigma_{i}\right]$,

$$
\begin{aligned}
& \left|\mathcal{I}_{\tau_{i}, \tau}^{\beta} h_{i}(\tau, x(\tau))-\mathcal{I}_{\tau_{i}, \tau}^{\beta} h_{i}(\tau, y(\tau))\right| \leq \frac{L_{h_{i}}}{\Gamma(\beta)} \int_{\tau_{i}}^{\tau}(\tau-\sigma)^{\beta-1} \sigma^{\gamma} \\
& \quad \times|x(\sigma)-y(\sigma)| d \sigma \leq \frac{L_{h_{i}}}{\Gamma(\beta)}\|x-y\|_{P B} \int_{\tau_{i}}^{\tau}(\tau-\sigma)^{\beta-1} \sigma^{\gamma} e^{\theta \sigma} d \sigma \\
& \leq \frac{L_{h_{i}}}{\Gamma(\beta)}\|x-y\|_{P B}\left(\int_{\tau_{i}}^{\tau}(\tau-\sigma)^{\sigma_{1}(\beta-1)} \sigma^{\sigma_{1} \gamma} d \sigma\right)^{\frac{1}{\sigma_{1}}}\left(\int_{\tau_{i}}^{\tau} e^{\theta \sigma_{1}^{*} \sigma} d \sigma\right)^{\frac{1}{\sigma_{1}^{*}}} \\
& \leq \frac{L_{h_{i}}}{\Gamma(\beta)}\|x-y\|_{P B}\left(\int_{\tau_{i}}^{\tau}(\tau-\sigma)^{\sigma_{1}(\beta-1)} \sigma^{\sigma_{1} \gamma} d \sigma\right)^{\frac{1}{\sigma_{1}}}\left(\int_{\tau_{i}}^{\tau} e^{\theta \sigma_{1}^{*} \sigma} d \sigma\right)^{\frac{1}{\sigma_{1}^{*}}} \\
& \leq \frac{L_{h_{i}}}{\Gamma(\beta)}\|x-y\|_{P B}\left(\int_{0}^{\tau}(\tau-\sigma)^{\sigma_{1}(\beta-1)} \sigma^{\sigma_{1} \gamma} d \sigma\right)^{\frac{1}{\sigma_{1}}} \frac{e^{\theta \tau}}{\left(\theta \sigma_{1}^{*}\right)^{\frac{1}{\sigma_{1}^{*}}}} \\
& \leq \frac{L_{h_{i}} \omega_{2}}{\Gamma(\beta)\left(\theta \sigma_{1}^{*}\right)^{\frac{1}{\sigma_{1}^{*}}}} e^{\theta \tau}\|x-y\|_{P B},
\end{aligned}
$$

where $\omega_{2}=\left(T^{\sigma_{1}(\beta-1)+\sigma_{1} \gamma+1} \mathbb{B}\left(\sigma_{1} \gamma+1, \sigma_{1}(\beta-1)+1\right)\right)^{1 / \sigma_{1}}$. Thus

$$
\left|\mathcal{I}_{\tau_{i}, \tau}^{\beta} h_{i}(\tau, x(\tau))-\mathcal{I}_{\tau_{i}, \tau}^{\beta} h_{i}(\tau, y(\tau))\right| e^{-\theta \tau} \leq \frac{L_{h_{i}} \omega_{2}}{\Gamma(\beta)\left(\theta \sigma_{1}^{*}\right)^{\frac{1}{\sigma_{1}^{*}}}}\|x-y\|_{P B}, \tau \in\left(\tau_{i}, \sigma_{i}\right] .
$$

By definition of an operator $T$ and using equations (3.10) and (3.11), for any $x, y \in P C(J, \mathbb{R})$, we have:

Case (i): For $\tau \in\left(\sigma_{i}, \tau_{i+1}\right], i=1,2, \ldots, m$,

$$
\begin{aligned}
& |T x(\tau)-T y(\tau)| e^{-\theta \tau} \leq \mathcal{I}_{\tau_{i}, \sigma_{i}}^{\beta}\left|h_{i}\left(\sigma_{i}, x\left(\sigma_{i}\right)\right)-h_{i}\left(\sigma_{i}, y\left(\sigma_{i}\right)\right)\right| e^{-\theta \tau} \\
& \quad+\mathcal{I}_{\sigma_{i}, \tau}^{\alpha}\left|f\left(\tau, x(\tau), \int_{\sigma_{i}}^{\tau} h(\sigma, x(\sigma)) d \sigma\right)-f\left(\tau, y(\tau), \int_{\sigma_{i}}^{\tau} h(\sigma, y(\sigma)) d \sigma\right)\right| e^{-\theta \tau} \\
& \leq\left(\frac{L_{h_{i}} \omega_{2}}{\Gamma(\beta)\left(\theta \sigma_{1}^{*}\right)^{\frac{1}{\sigma_{1}^{*}}}}+\omega_{1} \frac{M_{f}}{\Gamma(\alpha)\left(\theta \sigma^{*}\right)^{\frac{1}{\sigma^{*}}}}+\frac{N_{f} K_{h}}{\Gamma(\alpha+1)} \frac{\left(\tau_{i+1}-\sigma_{i}\right)^{\alpha+\frac{1}{2}}}{(2 \alpha+1)^{\frac{1}{2}}(2 \theta)^{\frac{1}{2}}}\right)\|x-y\|_{P B} .
\end{aligned}
$$


Case (ii): For $\tau \in\left(0, \tau_{1}\right]$,

$$
\begin{aligned}
& |T x(\tau)-T y(\tau)| e^{-\theta \tau} \\
& \leq \mathcal{I}_{0, \tau}^{\alpha}\left|f\left(\tau, x(\tau), \int_{0}^{\tau} h(\sigma, x(\sigma)) d \sigma\right)-f\left(\tau, y(\tau), \int_{0}^{\tau} h(\sigma, y(\sigma)) d \sigma\right)\right| e^{-\theta \tau} \\
& \leq\left(\omega_{1} \frac{M_{f}}{\Gamma(\alpha)\left(\theta \sigma^{*}\right)^{\frac{1}{\sigma^{*}}}}+\frac{N_{f} K_{h}}{\Gamma(\alpha+1)} \frac{\tau_{1}^{\alpha+\frac{1}{2}}}{(2 \alpha+1)^{\frac{1}{2}}(2 \theta)^{\frac{1}{2}}}\right)\|x-y\|_{P B} .
\end{aligned}
$$

Case (iii): For $\tau \in\left(\sigma_{i}, \tau_{i}\right], i=1,2, \ldots, m$,

$$
\begin{aligned}
|T x(\tau)-T y(\tau)| e^{-\theta \tau} & \leq\left|\mathcal{I}_{\tau_{i}, \tau}^{\beta} h_{i}(\tau, x(\tau))-\mathcal{I}_{\tau_{i}, \tau}^{\beta} h_{i}(\tau, y(\tau))\right| e^{-\theta \tau} \\
& \leq \frac{L_{h_{i}} \omega_{2}}{\Gamma(\beta)\left(\theta \sigma_{1}^{*}\right)^{1 / \sigma_{1}^{*}}}\|x-y\|_{P B} .
\end{aligned}
$$

By Cases (i)-(iii), we have

$$
\|T x-T y\|_{P B} \leq \mathcal{L}_{1}\|x-y\|_{P B}, \text { for any } x, y \in P C(J, \mathbb{R}),
$$

where

$$
\begin{aligned}
\mathcal{L}_{1}=\max \left\{\frac{L_{h_{i}} \omega_{2}}{\Gamma(\beta)\left(\theta \sigma_{1}^{*}\right)^{1 / \sigma_{1}^{*}}}+\omega_{1} \frac{M_{f}}{\Gamma(\alpha)\left(\theta \sigma^{*}\right)^{1 / \sigma^{*}}}\right. & \\
& \left.\quad+\frac{N_{f} K_{h}}{\Gamma(\alpha+1)} \frac{\left(\tau_{i+1}-\sigma_{i}\right)^{\alpha+1 / 2}}{(2 \alpha+1)^{1 / 2}(2 \theta)^{1 / 2}}: i=0,1, \ldots, m\right\} .
\end{aligned}
$$

By choosing sufficient large value of $\theta$, we get $\mathcal{L}_{1}<1$ and in this case $T$ is a contraction and hence $T$ has a unique fixed point, which is the unique solution of $(3.1)$.

\section{Bielecki-Ulam-Hyers stability}

We adopt the idea of Wang and Zang [16] to investigate the concepts of BieleckiUlams type stability for the class of nonlinear fractional order Volterra integrodifferential equation (1.1).

For any $\theta>0, \epsilon>0, \psi \geq 0, \varphi \in P C\left(J, \mathbb{R}_{+}\right)$is nondecreasing and $\alpha, \beta \in(0,1)$, consider the following inequalities:

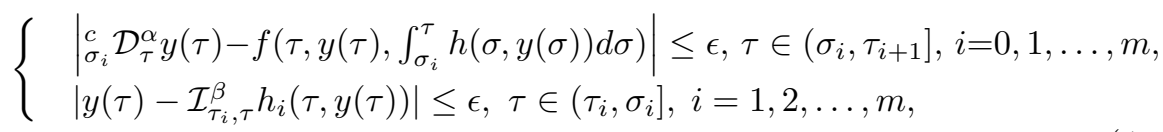

$$
\begin{aligned}
& \left\{\begin{array}{c}
\left|{ }_{\sigma_{i}}^{c} \mathcal{D}_{\tau}^{\alpha} y(\tau)-f\left(\tau, y(\tau), \int_{\sigma_{i}}^{\tau} h(\sigma, y(\sigma)) d \sigma\right)\right| \leq \varphi(\tau), \tau \in\left(\sigma_{i}, \tau_{i+1}\right], \\
i=0,1, \ldots, m, \\
\left|y(\tau)-\mathcal{I}_{\tau_{i}, \tau}^{\beta} h_{i}(\tau, y(\tau))\right| \leq \psi, \tau \in\left(\tau_{i}, \sigma_{i}\right], i=1,2, \ldots, m
\end{array}\right.
\end{aligned}
$$


and

$$
\left\{\begin{array}{c}
\mid \begin{array}{c}
{ }_{\sigma_{i}}^{c} \mathcal{D}_{\tau}^{\alpha} y(\tau)-f\left(\tau, y(\tau), \int_{\sigma_{i}}^{\tau} h(\sigma, y(\sigma)) d \sigma\right) \mid \leq \epsilon \varphi(\tau), \tau \in\left(\sigma_{i}, \tau_{i+1}\right] \\
i=0,1, \ldots, m \\
\left|y(\tau)-\mathcal{I}_{\tau_{i}, \tau}^{\beta} h_{i}(\tau, y(\tau))\right| \leq \epsilon \psi, \tau \in\left(\tau_{i}, \sigma_{i}\right], i=1,2, \ldots, m
\end{array}
\end{array}\right.
$$

Definition 3. The equation (1.1) is Bielecki-Ulam-Hyers stable if there exists a real number $C_{f, \theta, h, \alpha, \beta, h_{i}}>0$ such that for each $\epsilon>0$ for each solution $y \in P C^{1}(J, \mathbb{R})$ of inequality (4.1) there exists a solution $x \in P C^{1}(J, \mathbb{R})$ of equation (1.1) with

$$
|y(\tau)-x(\tau)| e^{-\theta \tau} \leq C_{f, \theta, h, \alpha, \beta, h_{i}} \epsilon, \quad \tau \in J .
$$

Definition 4. The equation (1.1) is generalized Bielecki-Ulam-Hyers stable if there exists $\theta_{f, \theta, h, \alpha, \beta, h_{i}} \in C\left(\mathbb{R}_{+}, \mathbb{R}_{+}\right), \theta_{f, \theta, h, \alpha, \beta, h_{i}}(0)=0$ such that for each $\epsilon>0$ for each solution $y \in P C^{1}(J, \mathbb{R})$ of inequality (4.1) there exists a solution $x \in P C^{1}(J, \mathbb{R})$ of equation (1.1) with

$$
|y(\tau)-x(\tau)| e^{-\theta \tau} \leq \theta_{f, \theta, h, \alpha, \beta, h_{i}}(\epsilon), \quad \tau \in J .
$$

Definition 5. The equation (1.1) is Bielecki-Ulam-Hyers-Rassias stable with respect to $(\phi, \psi)$ if there exists $C_{f, \theta, h, \alpha, \beta, h_{i}, \varphi}>0$ such that for each $\epsilon>0$ for each solution $y \in P C^{1}(J, \mathbb{R})$ of inequality (4.3) there exists a solution $x \in P C^{1}(J, \mathbb{R})$ of equation $(1.1)$ with

$$
|y(\tau)-x(\tau)| e^{-\theta \tau} \leq C_{f, \theta, h, \alpha, \beta, h_{i}, \varphi} \epsilon(\psi+\varphi(\tau)), \quad \tau \in J .
$$

Definition 6. The equation (1.1) is generalized Bielecki-Ulam-Hyers-Rassias stable with respect to $(\varphi, \psi)$ if there exists $C_{f, \theta, h, \alpha, \beta, h_{i}, \varphi}>0$ such that for each solution $y \in P C^{1}(J, \mathbb{R})$ of inequality (4.2) there exists a solution $x \in P C^{1}(J, \mathbb{R})$ of equation (1.1) with

$$
|y(\tau)-x(\tau)| e^{-\theta \tau} \leq C_{f, \theta, h, \alpha, \beta, h_{i}, \varphi}(\psi+\varphi(\tau)), \quad \tau \in J .
$$

Lemma 4. If $y \in P C^{1}(J, \mathbb{R})$ is a solution of inequality (4.3) then $y$ satisfies the following integral inequalities

$$
\left\{\begin{array}{l}
\left|y(\tau)-\mathcal{I}_{\tau_{i}, \tau}^{\beta} h_{i}(\tau, y(\tau))\right| \leq \epsilon \psi, \tau \in\left(\tau_{i}, \sigma_{i}\right], i=1,2, \ldots, m \\
\mid y(\tau)-y(0)-\mathcal{I}_{0, \tau}^{\alpha} f\left(\tau, y(\tau), \int_{0}^{\tau} h(\sigma, y(\sigma)) d \sigma \mid \leq \epsilon \mathcal{I}_{0, \tau}^{\alpha} \varphi(\tau), \text { if } \tau \in\left(0, \tau_{1}\right]\right. \\
\mid y(\tau)-\mathcal{I}_{\tau_{i}, \sigma_{i}}^{\beta} h_{i}\left(\sigma_{i}, y\left(\sigma_{i}\right)\right)-\mathcal{I}_{\sigma_{i}, \tau}^{\alpha} f\left(\tau, y(\tau), \int_{\sigma_{i}}^{\tau} h(\sigma, y(\sigma)) d \sigma \mid\right. \\
\leq \epsilon\left(\psi+\mathcal{I}_{\sigma_{i}, \tau}^{\alpha} \varphi(\tau)\right), \tau \in\left(\sigma_{i}, \tau_{i+1}\right], i=1,2, \ldots, m .
\end{array}\right.
$$

Proof. If $y \in P C^{1}(J, \mathbb{R})$ is a solution of the inequality (4.3) then there exists $H \in P C(J, \mathbb{R})$ and constants $H_{i}, i=1,2, \ldots, m$ (which depend on $y$ ) such that

(i) $|H(\tau)| \leq \epsilon \varphi(\tau), \tau \in J$ and $\left|H_{i}\right| \leq \epsilon \psi$ for $i=1,2, \ldots, m$. 
(ii) $\left\{\begin{array}{c}{ }_{\sigma_{i}}^{c} \mathcal{D}_{\tau}^{\alpha} y(\tau)=f\left(\tau, y(\tau), \int_{\sigma_{i}}^{\tau} h(\sigma, y(\sigma)) d \sigma\right)+H(\tau), \tau \in\left(\sigma_{i}, \tau_{i+1}\right], \\ i=0,1, \ldots, m, \\ y(\tau)=\mathcal{I}_{\tau_{i}, \tau}^{\beta} h_{i}(\tau, y(\tau))+H_{i}, \tau \in\left(\tau_{i}, \sigma_{i}\right], i=1,2, \ldots, m .\end{array}\right.$

In view of Lemma 3, above equation is equivalent to the integral equations

$$
y(\tau)=\left\{\begin{array}{l}
\mathcal{I}_{\tau_{i}, \tau}^{\beta} h_{i}(\tau, y(\tau))+H_{i}, \tau \in\left(\tau_{i}, \sigma_{i}\right], i=1, \ldots, m \\
y(0)+\mathcal{I}_{0, \tau}^{\alpha}\left[f\left(\tau, y(\tau), \int_{0}^{\tau} h(\sigma, y(\sigma)) d \sigma\right)+H(\tau)\right], \tau \in\left[0, \tau_{1}\right], \\
\mathcal{I}_{\tau_{i}, \sigma_{i}}^{\beta} h_{i}\left(\sigma_{i}, y\left(\sigma_{i}\right)\right)+H_{i}+\mathcal{I}_{\sigma_{i}, \tau}^{\alpha}\left[f\left(\tau, y(\tau), \int_{\sigma_{i}}^{\tau} h(\sigma, y(\sigma)) d \sigma\right)\right. \\
\quad+H(\tau)], \quad \tau \in\left(\sigma_{i}, \tau_{i+1}\right], i=1, \ldots, m .
\end{array}\right.
$$

For any $\tau \in\left(\sigma_{i}, \tau_{i+1}\right], i=1,2, \ldots, m$,

$$
\begin{aligned}
& \mid y(\tau)-\mathcal{I}_{\tau_{i}, \sigma_{i}}^{\beta} h_{i}\left(\sigma_{i}, y\left(\sigma_{i}\right)\right)-\mathcal{I}_{\sigma_{i}, \tau}^{\alpha} f\left(\tau, y(\tau), \int_{\sigma_{i}}^{\tau} h(\sigma, y(\sigma)) d \sigma \mid\right. \\
& =\left|H_{i}+\mathcal{I}_{\sigma_{i}, \tau}^{\alpha} H(\tau)\right| \leq\left|H_{i}\right|+\mathcal{I}_{\sigma_{i}, \tau}^{\alpha}|H(\tau)| \leq \epsilon\left(\psi+\mathcal{I}_{\sigma_{i}, \tau}^{\alpha} \varphi(\tau)\right) .
\end{aligned}
$$

For $\tau \in\left(0, \tau_{1}\right]$

$$
\mid y(\tau)-y(0)-\mathcal{I}_{0, \tau}^{\alpha} f\left(\tau, y(\tau), \int_{0}^{\tau} h(\sigma, y(\sigma)) d \sigma\left|=\mathcal{I}_{0, \tau}^{\alpha}\right| H(\tau) \mid \leq \epsilon \mathcal{I}_{0, \tau}^{\alpha} \varphi(\tau) .\right.
$$

For $\tau \in\left(\tau_{i}, \sigma_{i}\right], i=1,2, \ldots, m$,

$$
\left|y(\tau)-\mathcal{I}_{\tau_{i}, \sigma_{i}}^{\beta} h_{i}(\tau, y(\tau))\right| \leq\left|H_{i}\right| \leq \epsilon \psi, \tau \in\left(\tau_{i}, \sigma_{i}\right], i=1,2, \ldots, m .
$$

The last three inequalities are the required equivalent integral inequalities in (4.4).

Following additional hypothesis is needed to prove the Bielecki-Ulam-HyersRassias stability of equation (1.1).

(H4) Let $\varphi \in C\left(J, \mathbb{R}_{+}\right)$is nondecreasing and there exists $c_{\varphi}>0$ such that

$$
\mathcal{I}_{0, \tau}^{\alpha} \varphi \leq c_{\varphi} \varphi(\tau), \tau \in J
$$

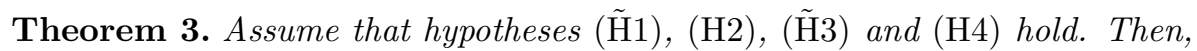
the equation (1.1) is Bielecki-Ulam-Hyers-Rassias stable with respect to $(\varphi, \psi)$, where $\alpha, \beta \in(0,1)$.

Proof. Let $y \in P C^{1}(J, \mathbb{R})$ be a solution of inequality (4.3). Then by Lemma 4, $y$ satisfies the integral inequalities

$$
\left\{\begin{array}{c}
\left|y(\tau)-\mathcal{I}_{\tau_{i}, \tau}^{\beta} h_{i}(\tau, y(\tau))\right| \leq \epsilon \psi, \tau \in\left(\tau_{i}, \sigma_{i}\right], i=1,2, \ldots, m \\
\mid y(\tau)-y(0)-\mathcal{I}_{0, \tau}^{\alpha} f\left(\tau, y(\tau), \int_{0}^{\tau} h(\sigma, y(\sigma)) d \sigma \mid \leq \epsilon c_{\varphi} \varphi(\tau), \tau \in\left(0, \tau_{1}\right]\right. \\
\mid y(\tau)-\mathcal{I}_{\tau_{i}, \sigma_{i}}^{\beta} h_{i}\left(\sigma_{i}, y\left(\sigma_{i}\right)\right)-\mathcal{I}_{\sigma_{i}, \tau}^{\alpha} f\left(\tau, y(\tau), \int_{\sigma_{i}}^{\tau} h(\sigma, y(\sigma)) d \sigma \mid\right. \\
\leq \epsilon\left(\psi+c_{\varphi} \varphi(\tau)\right), \quad \tau \in\left(\sigma_{i}, \tau_{i+1}\right], i=1,2, \ldots, m
\end{array}\right.
$$


Denote by $x$ the classical solution of fractional Volterra integrodifferential equations

$$
\left\{\begin{array}{l}
{ }_{\sigma_{i}}^{c} \mathcal{D}_{\tau}^{\alpha} x(\tau)=f\left(\tau, x(\tau), \int_{\sigma_{i}}^{\tau} h(\sigma, x(\sigma)) d \sigma\right), \tau \in\left(\sigma_{i}, \tau_{i+1}\right], i=0,1, \ldots, m \\
x(\tau)=\mathcal{I}_{\tau_{i}, \tau}^{\beta} h_{i}(\tau, x(\tau)), \tau \in\left(\tau_{i}, \sigma_{i}\right], i=1,2, \ldots, m \\
x(0)=y(0)
\end{array}\right.
$$

Then, in view of Lemma 3, $x$ satisfies the fractional Volterra integral equations

$$
x(\tau)=\left\{\begin{array}{l}
y(0), \tau=0, \\
\mathcal{I}_{\tau_{i}, \tau}^{\beta} h_{i}(\tau, x(\tau)), \tau \in\left(\tau_{i}, \sigma_{i}\right], i=1, \ldots, m \\
y(0)+\mathcal{I}_{0, \tau}^{\alpha} f\left(\tau, x(\tau), \int_{0}^{\tau} h(\sigma, x(\sigma)) d \sigma\right), \tau \in\left(0, \tau_{1}\right], \\
\mathcal{I}_{\tau_{i}, \sigma_{i}}^{\beta} h_{i}\left(\sigma_{i}, x\left(\sigma_{i}\right)\right)+\mathcal{I}_{\sigma_{i}, \tau}^{\alpha} f\left(\tau, x(\tau), \int_{\sigma_{i}}^{\tau} h(\sigma, x(\sigma)) d \sigma\right), \\
\tau \in\left(\sigma_{i}, \tau_{i+1}\right], i=1, \ldots, m .
\end{array}\right.
$$

Proceeding as in the proof of Theorem 2, for any $\tau \in\left(\sigma_{i}, \tau_{i+1}\right], i=1,2, \ldots, m$, we obtain

$$
\begin{aligned}
& |y(\tau)-x(\tau)| e^{-\theta \tau} \\
& =\left|y(\tau)-\left(\mathcal{I}_{\tau_{i}, \sigma_{i}}^{\beta} h_{i}\left(\sigma_{i}, x\left(\sigma_{i}\right)\right)+\mathcal{I}_{\sigma_{i}, \tau}^{\alpha} f\left(\tau, x(\tau), \int_{\sigma_{i}}^{\tau} h(\sigma, x(\sigma)) d \sigma\right)\right)\right| e^{-\theta \tau} \\
& \leq\left|y(\tau)-\mathcal{I}_{\tau_{i}, \sigma_{i}}^{\beta} h_{i}\left(\sigma_{i}, y\left(\sigma_{i}\right)\right)-\mathcal{I}_{\sigma_{i}, t}^{\alpha} f\left(\tau, y(\tau), \int_{\sigma_{i}}^{\tau} h(\sigma, y(\sigma)) d \sigma\right)\right| e^{-\theta \tau} \\
& \quad+\left|\mathcal{I}_{\sigma_{i}, \tau}^{\alpha} f\left(\tau, y(\tau), \int_{\sigma_{i}}^{\tau} h(\sigma, y(\sigma)) d \sigma\right)-\mathcal{I}_{\sigma_{i}, \tau}^{\alpha} f\left(\tau, x(\tau), \int_{\sigma_{i}}^{\tau} h(\sigma, x(\sigma)) d \sigma\right)\right| e^{-\theta \tau} \\
& =\epsilon\left(\psi+c_{\varphi} \varphi(\tau)\right) e^{-\theta \tau}+\frac{L_{h_{i}} \omega_{2}}{\Gamma(\beta)\left(\theta \sigma_{1}^{*}\right)^{\frac{1}{\sigma_{1}^{*}}}}\left(\sup _{\tau \in\left(\tau_{i}, \sigma_{i}\right]} e^{-\theta \tau}|y(\tau)-x(\tau)|\right) \\
& +\left[\frac{\omega_{1} M_{f}}{\Gamma(\alpha)\left(\theta \sigma^{*}\right)^{\frac{1}{\sigma^{*}}}}+\frac{N_{f} K_{h}\left(\tau_{i+1}-\sigma_{i}\right)^{\alpha+\frac{1}{2}}}{\Gamma(\alpha+1)(2 \alpha+1)^{\frac{1}{2}}(2 \theta)^{\frac{1}{2}}}\right]\left(\sup _{\tau \in\left(\sigma_{i}, \tau_{i+1}\right]} e^{-\theta \tau}|y(\tau)-x(\tau)|\right) .
\end{aligned}
$$

This gives

$$
\begin{aligned}
& \sup _{\tau \in\left(\sigma_{i}, \tau_{i+1}\right]} e^{-\theta \tau}|x(\tau)-y(\tau)| \leq \epsilon\left(1+c_{\varphi}\right)(\psi+\varphi(\tau)) e^{-\theta \sigma_{i}}+\left(\frac{L_{h_{i}} \omega_{2}}{\Gamma(\beta)\left(\theta \sigma_{1}^{*}\right)^{\frac{1}{\sigma_{1}^{*}}}}\right. \\
& \left.\quad+\frac{\omega_{1} M_{f}}{\Gamma(\alpha)\left(\theta \sigma^{*}\right)^{\frac{1}{\sigma^{*}}}}+\frac{N_{f} K_{h}\left(\tau_{i+1}-\sigma_{i}\right)^{\alpha+\frac{1}{2}}}{\Gamma(\alpha+1)(2 \alpha+1)^{\frac{1}{2}}(2 \theta)^{\frac{1}{2}}}\right) \sup _{\tau \in\left(\sigma_{i}, \tau_{i+1}\right]} e^{-\theta \tau}|x(\tau)-y(\tau)| .
\end{aligned}
$$

Therefore,

$$
\begin{aligned}
& \sup _{\tau \in\left(\sigma_{i}, \tau_{i+1}\right]} e^{-\theta \tau}|x(\tau)-y(\tau)| \\
& \leq \frac{\epsilon\left(1+c_{\varphi}\right)(\psi+\varphi(\tau)) e^{-\theta \sigma_{i}}}{1-\left(\frac{L_{h_{i}} \omega_{2}}{\Gamma(\beta)\left(\theta \sigma_{1}^{*}\right)^{\frac{1}{\sigma_{1}^{*}}}}+\frac{\omega_{1} M_{f}}{\Gamma(\alpha)\left(\theta \sigma^{*}\right)^{\frac{1}{\sigma^{*}}}}+\frac{N_{f} K_{h}\left(\tau_{i+1}-\sigma_{i}\right)^{\alpha+\frac{1}{2}}}{\Gamma(\alpha+1)(2 \alpha+1)^{\frac{1}{2}}(2 \theta)^{\frac{1}{2}}}\right)} .
\end{aligned}
$$


Now, for any $\tau \in\left(\tau_{i}, \sigma_{i}\right], i=1,2, \ldots, m$,

$$
\begin{aligned}
& |y(\tau)-x(\tau)| e^{-\theta \tau} \leq\left|y(\tau)-\mathcal{I}_{\tau_{i}, \tau}^{\beta} h_{i}(\tau, x(\tau))\right| e^{-\theta \tau} \\
& \quad \leq\left|y(\tau)-\mathcal{I}_{\tau_{i}, \tau}^{\beta} h_{i}(\tau, y(\tau))\right| e^{-\theta \tau}+\left|\mathcal{I}_{\tau_{i}, \tau}^{\beta} h_{i}(\tau, y(\tau))-\mathcal{I}_{\tau_{i}, \tau}^{\beta} h_{i}(\tau, x(\tau))\right| e^{-\theta \tau} \\
& \quad \leq \epsilon \psi e^{-\theta \tau_{i}}+\frac{L_{h_{i}} \omega_{2}}{\Gamma(\beta)\left(\theta \sigma_{1}^{*}\right)^{\frac{1}{\sigma_{1}^{*}}}}\left(\sup _{\tau \in\left(\tau_{i}, \sigma_{i}\right]} e^{-\theta \tau}|x(\tau)-y(\tau)|\right) .
\end{aligned}
$$

Therefore,

$$
\sup _{\tau \in\left(\tau_{i}, \sigma_{i}\right]} e^{-\theta \tau}|x(\tau)-y(\tau)| \leq \epsilon \psi e^{-\theta \tau_{i}}+\frac{L_{h_{i}} \omega_{2}}{\Gamma(\beta)\left(\theta \sigma_{1}^{*}\right)^{\frac{1}{\sigma_{1}^{*}}}}\left(\sup _{\tau \in\left(\tau_{i}, \sigma_{i}\right]} e^{-\theta \tau}|x(\tau)-y(\tau)|\right) .
$$

Thus, we have

$$
\sup _{\tau \in\left(\tau_{i}, \sigma_{i}\right]} e^{-\theta \tau}|x(\tau)-y(\tau)| \leq \frac{\epsilon \psi}{\left(1-L_{h_{i}} \omega_{2} /\left(\Gamma(\beta)\left(\theta \sigma_{1}^{*}\right)^{\frac{1}{\sigma_{1}^{*}}}\right)\right)} e^{-\theta \tau_{i}} .
$$

Next, for any $\tau \in\left(0, \tau_{1}\right]$,

$$
\begin{aligned}
& |y(\tau)-x(\tau)| e^{-\theta \tau} \leq\left|y(\tau)-y(0)-\mathcal{I}_{0, \tau}^{\alpha} f\left(\tau, x(\tau), \int_{0}^{\tau} h(\sigma, x(\sigma)) d \sigma\right)\right| e^{-\theta \tau} \\
& \leq\left|y(\tau)-y(0)-\mathcal{I}_{0, \tau}^{\alpha} f\left(\tau, y(\tau), \int_{0}^{\tau} h(\sigma, y(\sigma)) d \sigma\right)\right| e^{-\theta \tau} \\
& +\mathcal{I}_{0, \tau}^{\alpha}\left|f\left(\tau, y(\tau), \int_{0}^{\tau} h(\sigma, y(\sigma)) d \sigma\right)-f\left(\tau, x(\tau), \int_{0}^{\tau} h(\sigma, x(\sigma)) d \sigma\right)\right| e^{-\theta \tau} \\
& \leq \epsilon c_{\varphi} \varphi(\tau) \\
& +\left(\frac{\omega_{1} M_{f}}{\Gamma(\alpha)\left(\theta \sigma^{*}\right)^{\frac{1}{\sigma^{*}}}}+\frac{N_{f} K_{h}}{\Gamma(\alpha+1)} \frac{\tau_{1}^{\alpha+\frac{1}{2}}}{(2 \alpha+1)^{\frac{1}{2}}(2 \theta)^{\frac{1}{2}}}\right) \sup _{\tau \in\left(0, \tau_{1}\right]} e^{-\theta \tau}|x(\tau)-y(\tau)| .
\end{aligned}
$$

Just computed as above, we get

$$
\sup _{\tau \in\left(0, \tau_{1}\right]}|y(\tau)-x(\tau)| e^{-\theta \tau} \leq \frac{\epsilon c_{\varphi} \varphi(\tau)}{1-\left(\frac{\omega_{1} M_{f}}{\Gamma(\alpha)\left(\theta \sigma^{*}\right)^{\frac{1}{\sigma^{*}}}}+\frac{N_{f} K_{h}}{\Gamma(\alpha+1)} \frac{\tau_{1}^{\alpha+\frac{1}{2}}}{(2 \alpha+1)^{\frac{1}{2}}(2 \theta)^{\frac{1}{2}}}\right)} .
$$

Since

$$
J=(0, T]=\left[\bigcup_{i=0}^{m}\left(\sigma_{i}, \tau_{i+1}\right]\right]\left[\bigcup_{i=1}^{m}\left(\tau_{i}, \sigma_{i}\right]\right],
$$

from the inequalities $(4.5),(4.6)$ and (4.7), we obtain

$$
\begin{aligned}
& \sup _{\tau \in(0, T]}|y(\tau)-x(\tau)| e^{-\theta \tau} \leq \sup _{\tau \in\left(0, \tau_{1}\right]}|y(\tau)-x(\tau)| e^{-\theta \tau} \\
& +\sum_{i=1}^{m} \sup _{\tau \in\left(\sigma_{i}, \tau_{i+1}\right]}|y(\tau)-x(\tau)| e^{-\theta \tau}+\sum_{i=1}^{m} \sup _{\tau \in\left(\tau_{i}, \sigma_{i}\right]}|y(\tau)-x(\tau)| e^{-\theta \tau} \\
& \quad \leq \epsilon c_{f, \theta, h, \alpha, \beta, h_{i}, \varphi}(\psi+\varphi(\tau)),
\end{aligned}
$$


where

$$
\begin{aligned}
& c_{f, \theta, h, \alpha, \beta, h_{i}, \varphi}=\frac{c_{\varphi}}{1-\left(\frac{\omega_{1} M_{f}}{\Gamma(\alpha)\left(\theta \sigma^{*}\right)^{\frac{1}{\sigma^{*}}}}+\frac{N_{f} K_{h}}{\Gamma(\alpha+1)} \frac{\tau_{1}^{\alpha+\frac{1}{2}}}{(2 \alpha+1)^{\frac{1}{2}}(2 \theta)^{\frac{1}{2}}}\right)}+\sum_{i=1}^{m} \frac{\psi}{\left(1-\frac{L_{h_{i}} \omega_{2}}{\Gamma(\beta)\left(\theta \sigma_{1}^{*}\right)^{\frac{1}{\sigma_{1}^{*}}}}\right)} \\
&+\sum_{i=1}^{m} \frac{1+c_{\varphi}}{1-\left(\frac{L_{h_{i}} \omega_{2}}{\Gamma(\beta)\left(\theta \sigma_{1}^{*}\right)^{\frac{1}{\sigma_{1}^{*}}}}+\frac{\omega_{1} M_{f}}{\Gamma(\alpha)\left(\theta \sigma^{*}\right)^{\frac{1}{\sigma^{*}}}}+\frac{N_{f} K_{h}\left(\tau_{i+1}-\sigma_{i}\right)^{\alpha+\frac{1}{2}}}{\Gamma(\alpha+1)(2 \alpha+1)^{\frac{1}{2}}(2 \theta)^{\frac{1}{2}}}\right)} .
\end{aligned}
$$

Finally, from inequality (4.8), we have

$$
|y(\tau)-x(\tau)| e^{-\theta \tau} \leq \epsilon c_{f, \theta, h, \alpha, \beta, h_{i}, \varphi}(\psi+\varphi(\tau)), \tau \in J .
$$

This shows that equation (1.1) Bielecki-Ulam-Hyers-Rassias stable with respect to $(\varphi, \psi)$.

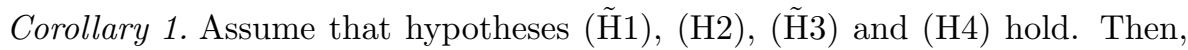
the equation (1.1) is generalized Bielecki-Ulam-Hyers-Rassias stable with respect to $(\varphi, \psi)$, where $\alpha, \beta \in(0,1)$.

Proof. Set $\epsilon=1$ in the proof of Theorem 2, we obtain

$$
|y(\tau)-x(\tau)| e^{-\theta \tau} \leq c_{f, \theta, h, \alpha, \beta, i, \varphi}(\psi+\varphi(\tau)), \tau \in J .
$$

This proves the equation (1.1) is generalized Bielecki-Ulam-Hyers-Rassias stable with respect to $(\varphi, \psi)$.

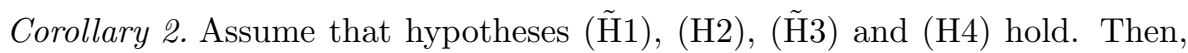
the equation (1.1) is Bielecki-Ulam-Hyers stable, where $\alpha, \beta \in(0,1)$.

Proof. Set $\psi=1$ and $\varphi(\tau)=1, \tau \in J$ in the proof of Theorem 2. Then $\varphi \in C\left(J, \mathbb{R}_{+}\right)$and $\mathcal{I}_{0, \tau}^{\alpha} \varphi \leq c_{\varphi} \varphi(\tau), \tau \in J$, where $c_{\varphi}=\frac{T^{\alpha}}{\Gamma(1+\alpha)}$. Thus the hypothesis (H4) is satisfied. Further, we have

$$
|y(\tau)-x(\tau)| e^{-\theta \tau} \leq \epsilon c_{f, \theta, h, \alpha, \beta, h_{i}}, \tau \in J .
$$

Therefore the equation (1.1) is Bielecki-Ulam-Hyers stable.

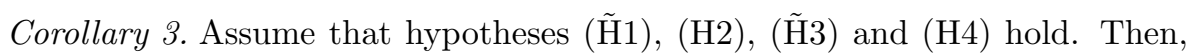
the equation (1.1) is generalized Bielecki-Ulam-Hyers stable, where $\alpha, \beta \in$ $(0,1)$.

Proof. Define $\theta_{f, \theta, h, \alpha, \beta, h_{i}}: \mathbb{R}_{+} \rightarrow \mathbb{R}_{+}$by $\theta_{f, \theta, h, \alpha, \beta, h_{i}}(\epsilon)=\epsilon c_{f, \theta, h, \alpha, \beta, h_{i}}$. Then $\theta_{f, \theta, h, \alpha, \beta, h_{i}} \in C\left(\mathbb{R}_{+}, \mathbb{R}_{+}\right)$and $\theta_{f, \theta, h, \alpha, \beta, h_{i}}(0)=0$. Further, from the equation (4.9), we have

$$
|y(\tau)-x(\tau)| e^{-\theta t} \leq \theta(\epsilon), \tau \in J,
$$

which shows that (1.1) is generalized Bielecki-Ulam-Hyers stable.

Remark 1. Under the hypotheses of Theorem 1, one can obtain the Bielecki Ulam-Hyers stability and Bielecki-Ulam-Hyers-Rassias stability of equation for $\alpha, \beta \in\left(\frac{1}{2}, 1\right)$. 


\section{Example}

Example 1. Consider Caputo fractional differential equation with fractional integrable impulse

$$
\begin{aligned}
& { }_{\sigma_{i}}^{c} \mathcal{D}_{\tau}^{\frac{2}{3}} x(\tau)=f\left(\tau, x(\tau), \int_{\sigma_{i}}^{\tau} h(\sigma, x(\sigma)) d \sigma\right), \quad \tau \in\left(\sigma_{i}, \tau_{i+1}\right], i=0,1, \\
& x(\tau)=\mathcal{I}_{\tau_{1}, \tau}^{\frac{2}{3}} h_{1}(\tau, x(\tau)), \quad \tau \in\left(\tau_{1}, \sigma_{1}\right] \\
& x(0)=0
\end{aligned}
$$

where $0=\sigma_{0}=\tau_{0}<\tau_{1}=1<\sigma_{1}=2<\tau_{2}=3$ and the functions $f$ : $[0,3] \times \mathbb{R} \times \mathbb{R} \rightarrow \mathbb{R} ; h:[0,3] \times \mathbb{R} \rightarrow \mathbb{R}$ and $h_{1}:(1,2] \times \mathbb{R} \rightarrow \mathbb{R}$ are defined as follows:

$$
\begin{aligned}
& f\left(\tau, x(\tau), \int_{\sigma_{i}}^{\tau} h(\sigma, x(\sigma)) d \sigma\right)= g(\tau)+\frac{e^{-\tau^{2}}}{4}(\sin (x(\tau))+\cos x(\tau)) \\
&+\int_{\sigma_{i}}^{\tau} \frac{\sigma}{e^{\sigma^{2}}} \sin (x(\sigma)) d \sigma \\
& h(\tau, x(\tau))=\frac{\tau}{e^{\tau^{2}}} \sin (x(\tau)), \quad h_{1}(\tau, x(\tau))=\frac{1}{\Gamma\left(\frac{4}{3}\right)} \frac{\tau(\tau-1)^{\frac{1}{3}}}{(\tau-4)}\left(\frac{|x(\tau)|-3}{|x(\tau)|+1}\right),
\end{aligned}
$$

where

$$
g(\tau)=\left\{\begin{array}{l}
\frac{9}{2 \Gamma(1 / 3)} \tau^{4 / 3}-\frac{1}{4}, \quad \tau \in[0,1] \\
0, \tau \in(1,2] \\
\frac{9}{2 \Gamma(1 / 3)}(\tau-2)^{4 / 3}-\frac{1}{4 e^{4}}(\cos 4+\sin 4), \quad \tau \in(2,3]
\end{array}\right.
$$

For any $x, y, \bar{x}, \bar{y} \in P C(J, \mathbb{R})$ and $\tau \in[0,3]$,

$$
\begin{aligned}
& |f(\tau, x, y)-f(\tau, \bar{x}, \bar{y})| \\
& =\left|\left(g(\tau)+\frac{e^{-\tau^{2}}}{4}(\sin x+\cos x)+y\right)-\left(g(\tau)+\frac{e^{-\tau^{2}}}{4}(\sin \bar{x}+\cos \bar{x})+\bar{y}\right)\right| \\
& \leq \frac{e^{-\tau^{2}}}{4}(|\sin x-\sin \bar{x}|+|\cos x-\cos \bar{x}|)+|y-\bar{y}|=\frac{e^{-\tau^{2}}}{4} \\
& \times\left(\left|2 \cos \left(\frac{x+\bar{x}}{2}\right) \sin \left(\frac{x-\bar{x}}{2}\right)\right|+\left|2 \sin \left(\frac{x+\bar{x}}{2}\right) \sin \left(\frac{x-\bar{x}}{2}\right)\right|\right)+|y-\bar{y}| \\
& \leq \frac{e^{-\tau^{2}}}{2}|x-\bar{x}|+|y-\bar{y}| \leq \frac{1}{2}|x-\bar{x}|+|y-\bar{y}| .
\end{aligned}
$$

Further, for $\tau \in[1,2]$,

$$
\begin{aligned}
& \left|h_{1}(\tau, x)-h_{1}(\tau, \bar{x})\right|=\left|\frac{1}{\Gamma\left(\frac{4}{3}\right)} \frac{\tau(\tau-1)^{\frac{1}{3}}}{(\tau-4)}\left(\frac{|x|-3}{|x|+1}\right)-\frac{1}{\Gamma\left(\frac{4}{3}\right)} \frac{\tau(\tau-1)^{\frac{1}{3}}}{(\tau-4)}\left(\frac{|y|-3}{|y|+1}\right)\right| \\
& \leq \frac{1}{\Gamma\left(\frac{4}{3}\right)}\left|\frac{|x|-3}{|x|+1}-\frac{|y|-3}{|y|+1}\right| \leq \frac{4}{\Gamma\left(\frac{4}{3}\right)} \frac{|| x|-| y||}{(|x|+1)(|y|+1)} \leq \frac{4}{\Gamma\left(\frac{4}{3}\right)}|x-y|
\end{aligned}
$$


and for $\tau \in[0,3]$, we have

$$
\begin{aligned}
& |h(\tau, x(\tau))-h(\tau, \bar{x})|=\left|\frac{\tau}{e^{\tau^{2}}} \sin x-\frac{\tau}{e^{\tau^{2}}} \sin \bar{x}\right|=\frac{\tau}{e^{\tau^{2}}}|\sin x-\sin \bar{x}| \\
& \quad=\frac{\tau}{e^{\tau^{2}}}|\sin x-\sin \bar{x}|=\frac{\tau}{e^{\tau^{2}}}\left|2 \cos \left(\frac{x+\bar{x}}{2}\right) \sin \left(\frac{x-\bar{x}}{2}\right)\right| \leq 3|x-\bar{x}| .
\end{aligned}
$$

Thus $f, h$ and $h_{1}$ satisfies Lipschitz condition with Lipschitz constants

$$
M_{f}=\frac{1}{2}, N_{f}=1, K_{h}=3 \text { and } L_{h_{1}}=\frac{4}{\Gamma(4 / 3)} .
$$

Thus by Theorem 1, the problem (5.1)-(5.3) has a unique solution on $[0,3]$. Let $\varphi(\tau)=2.8361 \mathbb{E}_{\frac{2}{3}}\left(\tau^{\frac{2}{3}}\right), \tau \in J$ and $\psi=0$. Then $\varphi \in C\left(J, \mathbb{R}_{+}\right)$is nondecreasing and satisfy the condition

$$
\mathcal{I}_{0, \tau}^{\frac{2}{3}} \varphi(\tau)=2.8361 \mathcal{I}_{\sigma_{i}, \tau}^{\frac{2}{3}}\left(\mathbb{E}_{\frac{2}{3}}\left(\tau^{\frac{2}{3}}\right)\right) \leq 2.8361 \mathbb{E}_{\frac{2}{3}}\left(\tau^{\frac{2}{3}}\right)=c_{\varphi} \varphi(\tau), \tau \in J,
$$

where $c_{\varphi}=1$. Note that all the hypotheses of the Corollary 1 hold. Therefore problem (5.1)-(5.2) is generalized Bielecki-Ulam-Hyers-Rassias stable with respect to $(\varphi, \psi)$.

Next, we shall discuss the generalized Bielecki-Ulam-Hyers-Rassias stablity of the equation (5.1)-(5.2) by showing that there exists an exact solution $x(\tau)$ of the problem $(5.1)-(5.3)$ corresponding to $(\varphi, \psi)$ and the given solution $y(\tau)$ of the inequalities

$$
\begin{aligned}
& ||_{\sigma_{i}}^{c} \mathcal{D}_{\tau}^{\frac{2}{3}} y(\tau)-f\left(\tau, y(\tau), \int_{\sigma_{i}}^{\tau} h(\sigma, y(\sigma)) d \sigma\right) \mid \leq \varphi(\tau), \tau \in\left(\sigma_{i}, \tau_{i+1}\right], i=0,1 \\
& \left|y(\tau)-\mathcal{I}_{\tau_{1}, \tau}^{\frac{2}{3}} h_{1}(\tau, y(\tau))\right| \leq 0, \quad \tau \in\left(\tau_{1}, \sigma_{1}\right]
\end{aligned}
$$

where $0=\sigma_{0}=\tau_{0}<\tau_{1}=1<\sigma_{1}=2<\tau_{2}=3$. Let

$$
y(\tau)=\left\{\begin{array}{l}
\tau, \tau \in[0,1] \cup(2,3] \\
(\tau-1), \tau \in(1,2] .
\end{array}\right.
$$

Then for $\tau \in(0,1]$,

$$
\begin{aligned}
& \left|{ }_{0}^{c} \mathcal{D}_{\tau}^{\frac{2}{3}} y(t)-g(\tau)-\frac{e^{-\tau^{2}}}{4}(\sin y(\tau)+\cos y(\tau))-\int_{0}^{\tau} \frac{\sigma}{e^{\sigma^{2}}} \sin y(\sigma) d \sigma\right| \\
& =\left.\right|_{0} ^{c} \mathcal{D}_{\tau}^{\frac{2}{3}}(\tau)-\frac{9}{2 \Gamma(1 / 3)} \tau^{\frac{4}{3}}+\frac{1}{4}-\frac{e^{-\tau^{2}}}{4}(\sin \tau+\cos \tau)-\int_{0}^{\tau} \frac{\sigma}{e^{\sigma^{2}}} \sin \sigma d \sigma \mid \\
& \leq \frac{1}{\Gamma\left(\frac{4}{3}\right)} \tau^{\frac{1}{3}}+\frac{9}{2 \Gamma\left(\frac{1}{3}\right)} \tau^{\frac{4}{3}}+\frac{1}{4}+\frac{e^{-\tau^{2}}}{4}(|\sin \tau|+|\cos \tau|)+\int_{0}^{\tau} \frac{\sigma}{e^{\sigma^{2}}}|\sin \sigma| d \sigma \\
& \leq 4.5495
\end{aligned}
$$


and for $\tau \in(2,3]$,

$$
\begin{aligned}
& \left.\right|_{2} ^{c} \mathcal{D}_{\tau}^{\frac{2}{3}} y(\tau)-g(\tau)-\frac{e^{-\tau^{2}}}{4}(\sin (y(\tau))+\cos y(\tau))-\int_{2}^{\tau} \frac{\sigma}{e^{\sigma^{2}}} \sin (y(\sigma)) d \sigma \mid \\
& =\left.\right|_{2} ^{c} \mathcal{D}_{\tau}^{\frac{2}{3}}[(\tau-2)+2]-\frac{9}{2 \Gamma(1 / 3)}(\tau-2)^{\frac{4}{3}}+\frac{1}{4 e^{4}}(\cos 4+\sin 4) \\
& \quad-\frac{e^{-\tau^{2}}}{4}(\sin \tau+\cos \tau)-\int_{2}^{\tau} \frac{\sigma}{e^{\sigma^{2}}} \sin \sigma d \sigma \mid \leq \frac{1}{\Gamma(4 / 3)}(\tau-2)^{\frac{1}{3}}+0 \\
& +\frac{9}{2 \Gamma(1 / 3)}(\tau-2)^{\frac{4}{3}}+\frac{1}{4 e^{4}}(|\cos 4|+|\sin 4|)+\frac{1}{4 e^{4}}(|\sin \tau|+|\cos \tau|) \\
& \quad+\int_{2}^{\tau} \frac{\sigma}{e^{\sigma^{2}}}|\sin \sigma| d \sigma \leq 2.8361 .
\end{aligned}
$$

Therefore, for $\tau \in\left(\sigma_{i}, \tau_{i}\right], i=0,1$, we have

$$
\begin{gathered}
\left.\right|_{\sigma_{i}} ^{c} \mathcal{D}_{\tau}^{\frac{2}{3}} y(\tau)-g(\tau)-\frac{e^{-\tau^{2}}}{4}(\sin y(\tau)+\cos y(\tau))-\int_{\sigma_{i}}^{\tau} \frac{\sigma}{e^{\sigma^{2}}} \sin y(\sigma) d \sigma \mid \\
\leq \min \{4.5495,2.8361\}=2.8361 \leq 2.8361 \mathbb{E}_{\frac{2}{3}}\left(\tau^{\frac{2}{3}}\right)=\varphi(\tau) .
\end{gathered}
$$

Also, for $\tau \in(1,2]$, we get

$$
\left|y(\tau)-\mathcal{I}_{1, \tau}^{\frac{2}{3}}\left(h_{1}(\tau, y(\tau))\right)\right|=|(\tau-1)-(\tau-1)|=0 .
$$

Hence, $y(\tau)$ is a solution of an inequality (5.4)-(5.5).

Next, one can easily verify that

$$
x(\tau)=\left\{\begin{array}{l}
\tau^{2}, \quad \tau \in(0,1] \cup(2,3], \\
(\tau-1), \quad \tau \in(1,2]
\end{array}\right.
$$

is the unique solution of the problem (5.1)-(5.3).

As discussed in the proof of the Theorem 1, we have

$$
\begin{gathered}
\mathcal{L}=\max \left\{\frac{L_{h_{i}}}{\Gamma(\beta)} \frac{\left(\sigma_{i}-\tau_{i}\right)^{\beta-\frac{1}{2}}}{\sqrt{(2 \beta-1)} \sqrt{2 \theta}}+\frac{M_{f}}{\Gamma(\alpha)} \frac{\left(\tau_{i+1}-\sigma_{i}\right)^{\alpha-\frac{1}{2}}}{\sqrt{(2 \alpha-1)} \sqrt{2 \theta}}\right. \\
\left.+\frac{N_{f} K_{h}}{\Gamma(\alpha+1)} \frac{\left(\tau_{i+1}-\sigma_{i}\right)^{\alpha+\frac{1}{2}}}{\sqrt{2 \alpha+1} \sqrt{2 \theta}} ; i=0,1\right\} \\
=\max \left\{\frac{M_{f}}{\Gamma(\alpha)} \frac{1}{\sqrt{(2 \alpha-1)} \sqrt{2 \theta}}+\frac{N_{f} K_{h}}{\Gamma(\alpha+1)} \frac{1}{\sqrt{\sqrt{2 \alpha+1}} \sqrt{2 \theta}},\right. \\
\frac{L_{h_{1}}}{\Gamma(\beta)} \frac{1}{\sqrt{(2 \beta-1)} \sqrt{2 \theta}}+\frac{M_{f}}{\Gamma(\alpha)} \frac{1}{\sqrt{(2 \alpha-1)} \sqrt{2 \theta}} \\
\left.+\frac{N_{f} K_{h}}{\Gamma(\alpha+1)} \frac{1}{\sqrt{2 \alpha+1} \sqrt{2 \theta}}\right\}=\frac{7.5188 \sqrt{3}}{\Gamma\left(\frac{2}{3}\right) \sqrt{2 \theta}} .
\end{gathered}
$$


Choose $\theta>\left(\frac{7.5188 \sqrt{3}}{\sqrt{2} \Gamma\left(\frac{2}{3}\right)}\right)^{2}=46.2473$, so that $\mathcal{L}<1$. For this choice of $\theta$, we have: for $\tau \in(0,1]$,

$$
|y(\tau)-x(\tau)| e^{-\theta \tau}=\left|\tau-\tau^{2}\right| e^{-\theta \tau} \leq\left(\tau+\tau^{2}\right) \leq 2,
$$

for $\tau \in(1,2]$,

$$
|y(\tau)-x(\tau)| e^{-\theta \tau}=0
$$

and for $\tau \in(2,3]$,

$$
|y(\tau)-x(\tau)| e^{-\theta \tau}=\left|\tau-\tau^{2}\right| e^{-\theta \tau} \leq\left(\tau+\tau^{2}\right) e^{-2 \theta}=11 e^{-2 \theta} .
$$

Thus,

$$
|y(\tau)-x(\tau)| e^{-\theta \tau} \leq C_{f, \theta, h, \alpha, \beta, h_{i}, \varphi}(\psi+\varphi(\tau)), \quad \tau \in J=[0,3],
$$

where $C_{f, \theta, h, \alpha, \beta, h_{i}, \varphi}=1, \psi=0$ and $\varphi(\tau)=2.8361 \mathbb{E}_{\frac{2}{3}}\left(\tau^{\frac{2}{3}}\right)$.

\section{References}

[1] D.D. Bainov, V. Lakshmikantham and P.S. Simeonov. Theory of impulsive differential equations, volume 6. Series in Modern Applied Mathematics: World Scientific, Singapore, 1989. https://doi.org/10.1142/0906.

[2] M. Benchora, J. Hendroson and S. Ntouyas. Impulsive differential equations and inclusions, volume 2. Contemporary mathematics and its applications, New York, NY, USA; Hindawi, 2006. https://doi.org/10.1155/9789775945501.

[3] N. Eghbali, V. Kalvandi and J.M. Rassias. A fixed point approach to the MittagLeffler-Hyers-Ulam stability of a fractional integral equation. Open Mathematics, 14:237-246, 2016. https://doi.org/10.1515/math-2016-0019.

[4] M. Frigon and D. O'Regan. Existence results for first order impulsive differential equations. J. Math. Anal. Appl., 193:96-113, 1995. https://doi.org/10.1006/jmaa.1995.1224.

[5] M. Frigon and D. O'Regan. Impulsive differential equations with variable time. Nonlinear Analysis: TMA, 26:9113-9122, 1996. https://doi.org/10.1016/0362546X(95)00053-X.

[6] M. Frigon and D. O'Regan. First order impulsive initial and periodic problems with variable moments. J. Math. Anal. Appl., 233(2):730-739, 1999. https://doi.org/10.1006/jmaa.1999.6336.

[7] I. Podlubny. Fractional differential equations. Academic Press, San Diego, 1999.

[8] A.P. Prudnikov, Y.A. Brychkov and O.I. Marichev. Integral and series, Elementary Functions, volume I. Nauka, Moscow, 1981.

[9] A.M. Samoilenko and N.A. Perestyuk. Impulsive differential equations, volume 14. World Scientific series on nonlinear science, Singapore, 1995. https://doi.org/10.1142/9789812798664.

[10] S.M. Ulam. A collection of the mathematical problems. Interscience Publ., New York, 1960. 
[11] J. Wang and X. Li. $e_{\alpha}$-Ulam type stability of fractional order ordinary differential equations. J. Appl. Math. Comput., 45:449-459, 2014. https://doi.org/10.1007/s12190-013-0731-8.

[12] J. Wang, Z. Lin and Y. Zhou. On the stability of new impulsive ordinary differential equations. Topological Methods in Nonlinear Analysis, 46(1):303-314, 2015. https://doi.org/10.12775/TMNA.2015.048.

[13] J. Wang, L. Lv and Y. Zhou. Ulam stability and data dependence for fractional differential equations with Caputo derivative. Qualit. Th. Diff. Equat., 63:1-10, 2011. https://doi.org/10.14232/ejqtde.2011.1.63.

[14] J. Wang, L. Lv and Y. Zhou. New concepts and results in stability of fractional differential equations. Commun. Nonlinear Sci. Numer. Simulat., 17:2530-2538, 2012. https://doi.org/10.1016/j.cnsns.2011.09.030.

[15] J. Wang, A. Zada and W. Ali. Differential equations with variable delay in quasi-Banach spaces. Int. J. Nonlinear Sci. Num., 19(5):553-560, 2018. https://doi.org/10.1515/ijnsns-2017-0245.

[16] J. Wang and Y. Zhang. A class of nonlinear differential equations with fractional integrable impulses. Commun. Nonlinear Sci. Numer. Simulat., 19:3001-3010, 2014. https://doi.org/10.1016/j.cnsns.2014.01.016.

[17] J. Wang and Y. Zhang. Existence and stability of solutions to nonlinear impulsive differential equations in $\beta$-normed spaces. Electronic J. Differential Equations, 83:1-10, 2014.

[18] J. Wang and Y. Zhang. Ulam-Hyers-Mittag-Leffler stability of fractionalorder delay differential equations. Optimization, 63(8):1181-1190, 2014. https://doi.org/10.1080/02331934.2014.906597.

[19] Wei Wei, Xuezhu Li and Xia Li. New stability results for fractional integral equation. Computers and Mathematics with Applications, 64:3468-3476, 2012. https://doi.org/10.1016/j.camwa.2012.02.057.

[20] X.Wang, M. Arif and A. Zada. $\beta$-Hyers-Ulam-Rassias stability of semilinear nonautonomous impulsive system. Symmetry, 11(2):231, 2019. https://doi.org/10.3390/sym11020231.

[21] A. Zada and S. Ali. Stability analysis of multi-point boundary value problem for sequential fractional differential equations with noninstantaneous impulses. Int. J. Nonlinear Sci. Numer. Simul., 19(7):763-774, 2018. https://doi.org/10.1515/ijnsns-2018-0040.

[22] A. Zada, W. Ali and C. Park. Ulam's type stability of higher order nonlinear delay differential equations via integral inequality of Gronwall-Bellman-Bihari's type. Appl. Math. Comput., 350:60-65, 2019. https://doi.org/10.1016/j.amc.2019.01.014.

[23] A. Zada and S.O. Shah. Hyers-Ulam stability of first-order non-linear delay dfferential equations with fractional integrable impulses. Hacettepe J. Math. Stat., 47(5):1196-1205, 2018. https://doi.org/10.15672/HJMS.2017.496.

[24] A. Zada, S. Shaleena and T. Li. Stability analysis of higher order nonlinear dfferential equations in $\beta$-normed spaces. Math. Meth. App. Sci., 42(4):11511166, 2019. https://doi.org/10.1002/mma.5419.

[25] A. Zada, M. Yar and T. Li. Existence and stability analysis of nonlinear sequential coupled system of Caputo fractional differential equations with integral boundary conditions. Ann. Univ. Paedagog. Crac. Stud. Math., 417:103-125, 2018. https://doi.org/10.2478/aupcsm-2018-0009. 